Review

\title{
Understanding the Effects of Gut Microbiota Dysbiosis on Nonalcoholic Fatty Liver Disease and the Possible Probiotics Role: Recent Updates
}

\author{
Ashiq Khan ${ }^{1,2}$, Zitong Ding1 ${ }^{\bowtie}$, Muhammad Ishaq ${ }^{1}$, Ali Sher Bacha1, Israr Khan ${ }^{3}$, Anum Hanif1, Wenyuan \\ $\mathrm{Li}^{1}$, and Xusheng Guo ${ }^{1 凶}$ \\ 1. School of Life Sciences, Probiotics and Biological Feed Research Centre, Lanzhou University, Lanzhou 730000, PR China. \\ 2. Department of Microbiology, Balochistan University of Information Technology Engineering \& Management Sciences Quetta 87300, Pakistan. \\ 3. School of Life Sciences, Institute of Microbiology Lanzhou University, Lanzhou 730000, PR China. \\ $\bowtie$ Corresponding authors: Professor Xusheng Guo, Zitong Ding, E-mail: guoxsh07@lzu.edu.cn, dingwr@lzu.edu.cn, Tel: 86-931 13 8915650, Fax: 86 931 8915650.
}

(1) The author(s). This is an open access article distributed under the terms of the Creative Commons Attribution License (https://creativecommons.org/licenses/by/4.0/). See http://ivyspring.com/terms for full terms and conditions.

Received: 2020.11.20; Accepted: 2020.12.24; Published: 2021.02.08

\begin{abstract}
Nonalcoholic fatty liver disease (NAFLD) is leading chronic liver syndrome worldwide. Gut microbiota dysbiosis significantly contributes to the pathogenesis and severity of NAFLD. However, its role is complex and even unclear. Treatment of NAFLD through chemotherapeutic agents have been questioned because of their side effects on health. In this review, we highlighted and discussed the current understanding on the importance of gut microbiota, its dysbiosis and its effects on the gut-liver axis and gut mucosa. Further, we discussed key mechanisms involved in gut dysbiosis to provide an outline of its role in progression to NAFLD and liver cirrhosis. In addition, we also explored the potential role of probiotics as a treatment approach for the prevention and treatment of NAFLD. Based on the latest findings, it is evident that microbiota targeted interventions mostly the use of probiotics have shown promising effects and can possibly alleviate the gut microbiota dysbiosis, regulate the metabolic pathways which in turn inhibit the progression of NAFLD through the gut-liver axis. However, very limited studies in humans are available on this issue and suggest further research work to identify a specific core microbiome association with NAFLD and to discover its mechanism of pathogenesis, which will help to enhance the therapeutic potential of probiotics to NAFLD.
\end{abstract}

Key words: Nonalcoholic fatty liver disease; nonalcoholic steatohepatitis; gut microbiota; probiotics; dysbiosis; small intestinal bacterial overgrowth.

\section{Introduction}

Nonalcoholic fatty liver disease (NAFLD), the most prevalent liver disease, which is portrayed by the deposition of fats in the hepatocytes that ranges from simple hepatosteatosis to nonalcoholic steatohepatitis, fibrosis, cirrhosis, as well as hepatocellular carcinoma [1,2]. Currently, NAFLD is becoming a global health concern which accounts for $25 \%$ prevalence worldwide and more than $30 \%$ prevalence is projected among the adults by 2030, due to the dominance of obesity, unhealthy dietary patterns and sedentary ways of lifestyle [3-5]. Its incidence rate has increased with non-communicable infections, including cardiovascular disease, type 2 diabetes, as well as complex liver infections such as hepatic cirrhosis [6,7].

Historically, 'two-hit theory' was proposed to explain the pathogenesis of NAFLD, which revealed that oxidative stress is the triggering factor of hepatic injuries which develops from the simple steatosis to nonalcoholic steatohepatitis (NASH) followed by severe liver damage and cirrhosis [8,9]. However, studies in the recent decades have highlighted multiple risk factors, including gut microbiota dysbiosis, genetic, unhealthy diet, metabolic, insulin resistance (IR), environmental, host-derived features like age, ethnicity, gender, antibiotic use, alcohol 
consumption, oxidative stress, inflammatory states, lifestyle and so on. All these factors significantly contribute to NAFLD and hepatic steatosis and several molecular pathways have been identified in the progression of NAFLD [10-14]. Among these abnormal conditions, alteration of gut microbial populations has been recognized as the most common risk factor for NAFLD, obesity, and diabetes [15-17]. Further, NAFLD patients have found with higher dominance of small intestinal bacterial overgrowth in the gut [18], and this leads to gut dysbiosis [19].

Recent findings have shown that dysbiosis of gut microbiota also disturbs the hepatic carbohydrate, lipid metabolism and affects the balance among the pro and anti-inflammatory effectors in the liver, thus prompting NAFLD and its development to NASH [2]. However, the comprehensive mechanism of this severe liver disease is so far mysterious. Numerous new features remain yet to be revealed particularly to the specific functions of the gut microbiota [20,21]. Resolving these issues would be a milestone for the treatment of NAFLD and other gut microbiota related syndromes. Therefore, it is necessary to completely recognize the pathogenesis of this disease and the role of gut microbiota in its progression, which would possibly paw the way to discover new therapeutic strategies for control of NAFLD [2].

There is a growing interest in healthy gut microbiota and the restoration of dysbiosis gut microbiota. The probiotics are commonly used as an appealing approach due to their long traditional use, health-promoting properties and enormous literature supports. Evidence suggests that probiotics could regulate intestinal microbiota and can be preferred as a novel preventing or treatment approach for NAFLD and other chronic liver diseases [22,23]. Several latest findings have shown that probiotics have the tendency to change the dysbiosis gut microbiota to the normal and could be a potential treatment approach for the management of NAFLD [24-26]. As there is a close anatomical and functional connection among the gut and liver as well as microbial dysbiosis ensues NAFLD and probiotics could modulate this dysbiosis. Therefore, in this review we discussed the updated literature, significance of gut microbiota, its modulation and major proposed mechanisms of pathogenesis in dysbiosis gut and its progression to NAFLD. In addition, we also reviewed the role of most commonly used probiotics for the management of NAFLD.

\section{How Gut Microbiota Modulations Links in Progression of NAFLD?}

Gut microbiota is a complex ecosystem of microbes that lives throughout the gastrointestinal tract. It is the main site of microbial colonization and a diverse biome which consists of approximately 2,000 distinct bacterial species and collectively has a genome of about 150 fold more genes than in the human genome. Majority of the human gastrointestinal bacteria are composed of phyla Bacteroidetes, Firmicutes, Proteobacteria, Fusobacteria, Actinobacteria and Verrucomicrobia and Cyanobacteria [27]. Generally, Firmicutes, Actinobacteria, Bacteroidetes, Ruminococcaceae, Verrucomicrobia and Proteobacteria are more dominated in the gut $[28,29]$, and in a study authors found that healthy individual fecal accounts for about $80-90 \%$ of bacterial community [29]. Altogether these microbes have been developed along with the human [30].

Research has explored the composition of gut microbiota in patients with NAFLD relative to healthy individuals (Table 1), and identified the trends that can be associated with the development of NAFLD $[10,14]$. This shows that gut microbiota composition is adversely affected in patients with NAFLD. Gut microbiota in healthy individuals mostly has a balance among the microbiota, which perform vital functions, such as immune system regulation and detoxification. However, in NAFLD and other gut diseases, this balance among the major phyla disrupts (dysbiosis). Many of these bacterial groups of gut microbiota have been shown to either increase or decrease in NAFLD and produced various toxic metabolites, inflammation, oxidative stress and ultimately leads to liver cirrhosis.

In recent years, advances in technology have shown that human gut microbiota is extremely variable in abundance and composition and play significant role in supporting the human health and more varying in perspective of altered conditions (i.e. dietary, environmental and immune) which can lead to development of many diseases like obesity, NAFLD and hepatocellular carcinoma [4, 31-33].

Relatively higher abundance of genera, Fusobacteria, lower abundance of Oscillospira and Ruminococcus of Ruminococcaceae and Coprococcus of Lachnospiraceae, have been shown in NAFLD patients [34]. Further bacterial species were identified in NAFLD patients, including Proteobacteria, Escherichia and Enterobacteria [11], also Bacteroides were more in NASH patients paralleled to healthy individuals [19]. Similarly, authors in a study have shown a lower diversity of microbiota in feces of children infected with NAFLD and an increased number of Prevotellacopri and a lowest a- bacterial diversity was linked with severe liver fibrosis parallel to control [16]. 
Table 1. Impacts of NAFLD on the composition of gut microbiota

\begin{tabular}{|c|c|c|c|c|c|c|c|}
\hline \multirow[t]{2}{*}{ Human } & \multirow[t]{2}{*}{ Animal } & \multicolumn{5}{|c|}{ Gut Microbiota Composition in NAFLD } & \multirow[t]{2}{*}{ [Ref: } \\
\hline & & Phylum $(\uparrow / \downarrow)$ & Class & Family $(\uparrow / \downarrow)$ & Genus $(\uparrow / \downarrow)$ & Species $(\uparrow / \downarrow)$ & \\
\hline$\sqrt{ }$ & & $\begin{array}{l}\uparrow \text { Actinobacteria } \uparrow \text { Bacteroidetes } \\
\uparrow \text { Firmicutes } \\
\uparrow \text { Proteobacteria }\end{array}$ & & & $\uparrow$ Escherichia & & {$[11]$} \\
\hline$\sqrt{ }$ & & $\downarrow$ Bacteroidetes & & & & $\uparrow$ Clostridium coccoides & [37] \\
\hline & $\sqrt{ }$ & & & & & $\downarrow$ Akkermansia muciniphila & {$[38]$} \\
\hline$\sqrt{ }$ & & $\begin{array}{l}\uparrow \text { Firmicutes } \\
\uparrow \text { Proteobacteria }\end{array}$ & & $\uparrow$ Peptostreptococcaceae & $\begin{array}{l}\uparrow \text { Escherichia } \\
\uparrow \text { Lactobacillus } \\
\uparrow \text { Streptococcus } \\
\uparrow \text { Anaerobacter }\end{array}$ & & [39] \\
\hline$\sqrt{ }$ & & $\uparrow$ Bacteroides & & & $\begin{array}{l}\uparrow \text { Ruminococcus } \\
\downarrow \text { Prevotella }\end{array}$ & & [19] \\
\hline & $\sqrt{ }$ & $\begin{array}{l}\uparrow \text { Bacteroides } \\
\uparrow \text { Firmicutes }\end{array}$ & & & $\begin{array}{l}\uparrow \text { Atopobium } \\
\uparrow \text { Desulfovibrio }\end{array}$ & $\begin{array}{l}\uparrow \text { Bacteroides acidifaciens } \\
\uparrow \text { Clostridium cocleatum }\end{array}$ & {$[40]$} \\
\hline$\sqrt{ }$ & & $\uparrow$ Proteobacteria & & $\begin{array}{l}\uparrow \text { Lachnospiraceae } \\
\uparrow \text { Enterobacteriaceae }\end{array}$ & $\begin{array}{l}\uparrow \text { Fusobacteria } \\
\downarrow \text { Prevotella } \\
\uparrow \text { Blautia } \\
\uparrow \text { Escherichia } \\
\uparrow \text { Shigella }\end{array}$ & & [41] \\
\hline$\sqrt{ }$ & & $\begin{array}{l}\uparrow \text { Actinobacteria } \\
\downarrow \text { Bacteroidetes }\end{array}$ & & $\downarrow$ Rikenellaceae & $\begin{array}{l}\uparrow \text { Bradyrhizobium } \\
\uparrow \text { Anaerococcus } \\
\uparrow \text { Peptoniphilus } \\
\uparrow \text { Ruminococcus } \\
\downarrow \text { Oscillopira } \\
\downarrow \text { Rikenellaceae }\end{array}$ & $\uparrow$ Propioni bacterium acnes & [42] \\
\hline$\sqrt{ }$ & & $\uparrow$ Bacteroides & & & $\begin{array}{l}\uparrow \text { Lactobacilli } \\
\downarrow \text { Ruminococcu } \\
\downarrow \text { Bifidobacterium } \\
\uparrow \text { Prevotella } \\
\downarrow \text { Faecalibacterium }\end{array}$ & & {$[43]$} \\
\hline$\sqrt{ }$ & & & & & $\begin{array}{l}\uparrow \text { Lactobacilli } \\
\downarrow \text { Bifidobacteria }\end{array}$ & $\begin{array}{l}\uparrow \text { L. mucosae } \\
\downarrow \text { B.longum } \\
\downarrow \text { B.adolescent } \\
\downarrow \text { B.bifidum }\end{array}$ & [33] \\
\hline$\sqrt{ }$ & & $\uparrow$ Firmicutes $\uparrow$ Bacteroidetes & & $\uparrow$ Proteobacteria & & $\uparrow E$. coli & {$[44]$} \\
\hline$\sqrt{ }$ & & $\uparrow$ Bacteroides & & $\begin{array}{l}\uparrow \text { Enterobacteriaceae } \\
\uparrow R \text { Rminococcaceae }\end{array}$ & $\begin{array}{l}\uparrow \text { Streptococcus } \\
\downarrow \text { Akkermansia } \\
\downarrow \text { Bifidobacterium }\end{array}$ & & [45] \\
\hline$\sqrt{ }$ & & $\downarrow$ Firmicutes & & $\begin{array}{l}\downarrow \text { Desulfovibrionaceae } \\
\downarrow \text { Leuconostocaceae }\end{array}$ & $\begin{array}{l}\downarrow \text { Weissella } \\
\downarrow \text { Lactobacilli }\end{array}$ & & [46] \\
\hline
\end{tabular}

In addition, latest published study identified a decrease in alpha-microbial diversity and beta microbial diversity in a moderate stage of nonalcoholic fatty liver disease. Interestingly, they observed a decline in alpha $(\alpha)$ and the rise in beta $(\beta)$ microbial diversity in the severe stage of this disease (NAFLD-cirrhosis) [35]. Likewise, a recent study showed a higher proportion of Bacteroides as compared to the Lactobacillus as well as Bifidobacteria in the gut of the liver fibrosis patients [36]. These evidences shows that gut microbiota modulation may have a significant role in the development of NAFLD. Continuous advances in sequencing tools, as well as phonemic research now make this feasible to give a more comprehensive detailed functional dimensions of gut microbiota and its effects on the host liver phenotype [35]. Thereby, alterations of the microbial population in the gastrointestinal tract undoubtedly have potential effects on pathophysiology of gut diseases and studies are essential to discover innovative probiotics therapies that can restore the normal gut microbiota.

\section{Gut-liver axis Malfunction and its Impacts on NAFLD?}

The gut-liver axis is a close anatomical as well as the functional connection of the gastrointestinal tract (GIT) and liver. This axis is explained to increase the connections among the metabolites of the gut microbiome and liver surface receptors, which can activate a cascade of the severe events, leading to insulin resistance (IR), liver inflammation and enhanced liver fibrosis [47]. There is unique co-ordination among the gut and liver. Disruption of this axis has shown to play an essential role in the pathogenesis of several liver infections including NAFLD. The disruption includes gut barrier disturbance, bacterial translocation (BT) and inflammatory reactions in the liver, for example, Toll-like receptor signaling, activation of 
inflammasome as well as alterations in the composition of bacterial metabolites [2,48,49]. However, it is needed to clarify that this association is whether causal and further to elucidate the mechanism through which gut dysbiosis plays a role in the pathogenesis of NAFLD [50]. The altered gut microbes produce ammonia, ethanol and acetaldehyde and these toxic products may affect the liver functions via endotoxin release from Gram-negative bacteria or liver metabolism [48].

Cross talk among this axis is progressively recognized and reinforced via the correspondent increase in the incidence of immune disorders, gastrointestinal and liver diseases [51]. It is explored that gut microbiota dysbiosis and impairment of gut mucosal barrier affect the cross-talk between the gut and liver via small intestine bacterial outgrowth, which causes intestinal inflammation and could result in NAFLD [39]. It is also proposed that an obese individual gut microbiome can increase the pathogenesis of NAFLD by affecting lipid metabolism as well as enlarged its capacity to harvest more energy from the diet $[9,17]$. Therefore, due to these fluctuating features, modification of gut microbiota has produced a huge potential interest in the use of novel probiotics specifically for the prevention and treatment of chronic NAFLD [48]. Advances in technologies as well as understanding the research area of the gut-liver axis could boost research into gut microbiome based therapeutic approach to improve the management of this disease [52]. Consequently, studies are essential to address the link between the gut-liver axis and NAFLD and to elevate the role of potential probiotics and its mechanism of action on the gut liver axis for governing of NAFLD.

\section{The Links between Small Intestinal Bacterial Overgrowth and Leaky Gut and NAFLD Pathogenesis}

Small intestinal bacterial overgrowth (SIBO) is defined as an imbalance of normal gut microbiota in the small intestine [53]. This causes the disruption of gut microbiome diversity, its composition and associated with progression to NAFLD and other intestinal as well as extra intestinal diseases $[13,17,50]$. Among many potential contributions of gut microbes to liver diseases, small intestinal bacterial overgrowth (SIBO) has been comprehensively studied. Malfunction of gut microbiota can cause intense systematic infections [54]. Especially, dysbiotic microbes are often identified among the obese individuals and considered as a key risk factor for the development of NAFLD $[17,24]$. In dysbiosis and leaky gut, bacterial derived metabolic products (e.g. lipopolysaccharide) could induce inflammation of adipose tissues, hepatic steatosis, as well as hepatic inflammation $[55,56]$.

Gut dysbiosis is associated with the pathogenesis of NAFLD and its severity is connected with a larger abundance of inflammatory genes encoding bacterial products. Variations in the intestinal microbiome can contribute to the pathogenesis of NAFLD and can be used as biomarkers of disease and its severity [16]. Both NAFLD and obesity are associated with a larger concentration of Gram-negative bacterial species in the gut [11]. Gut leakiness is commonly caused by dysfunction of the intestinal barrier structures, which prompted intestinal inflammation as well as TNFR-I (TNF receptor-1) signaling in epithelial cells and it is showed that because of increased gut permeability, endotoxin, metabolic products, translocate into the liver [57], and activate the immune cells (Kupffer cells) via the receptors (TLR4 or TLR9). Then these cells produce various inflammatory cytokines and worsen the disease [58]. The role of other TLRs in the regulation of NAFLD remains mostly unknown, so it needs to be studied and further better understanding of tool- like receptors (TLR) signaling pathways in liver, which will help to explain the mechanisms of the liver tumorigenesis and will propose novel therapeutic targets for hepatocellular carcinoma [2].

Animal model investigations have evaluated the significance of gut dysbiosis in the development of NAFLD. Studies also determined that translocation of dysbiotic microbiota deteriorated the hepatic steatosis and therefore increased the progression of NAFLD $[11,37]$. A recent study has shown that gut microbiota from the obese human donors induced hepatic steatosis in germ-free mice via influencing lipid metabolism as well as transcriptional profile [59]. Authors in a study determined, that germ-free mice fed with high-fat diet were inoculated with gut microbes of NASH patients, showed a worsened $\mathrm{NASH}$ phenotype, as indicated by increased hepatic steatosis as well as inflammation [60]. In dysbiosis, the host is compromised and microbiota is unable to control the normal homeostasis and increase the intestinal permeability. These changes expose the liver to various exogenous and endogenous antigens through the gut-liver axis [61]. It is shown that SIBO might increase the absorption of endotoxins which initiate the pro-inflammatory as well as pro-fibrogenic effects on the liver [62]. Moreover, unusual changes like increased peroxidation of lipid, as well as oxidative stress, are effective in the raise of bacterial translocation (BT) [63]. Evidence also showed that compromised antimicrobial defense mechanisms could increase BT, results in portal hypertension and liver cirrhosis [64]. 


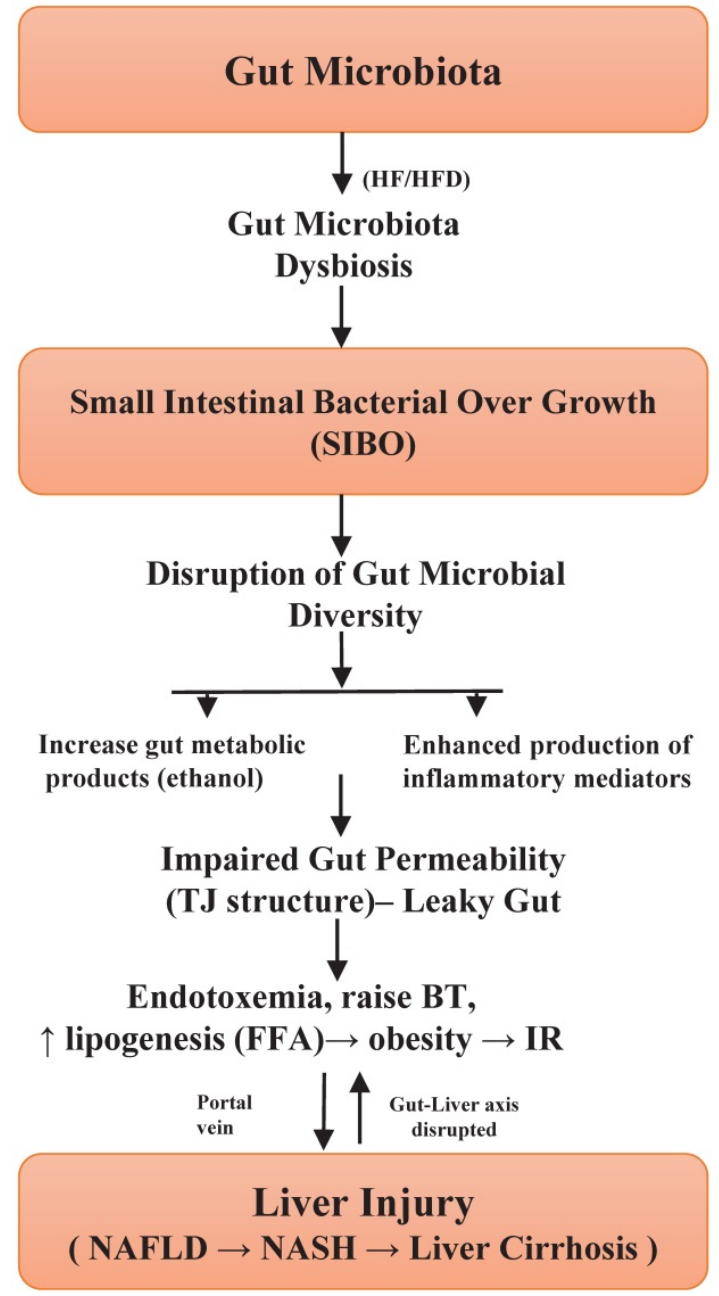

Figure 1. Illustration of how small intestinal bacterial overgrowth impacts the gut microbiota dysbiosis and progression of NAFLD.

Tight junction's proteins are mucosal barriers that inhibit the paracellular translocation of bacteria. Impairment in the integrity of this protein leads to an increase in the translocation of microbial metabolic products, for example, lipopolysaccharide (LPS) in the bloodstream and ensuing endotoxemia, can persuade inflated intestinal permeability and subsequently liver inflammation $[39,65]$. In addition, alterations in the intestinal tight junction structure have been studied in liver cirrhosis patients, however, the exact pathophysiology and dysfunction of the gut barrier are unclear. The maintenance of tight junction integrity would be a good approach to prevent or treat NAFLD [66], and other gut diseases. A study conducted in humans showed a significant relationship between gut permeability, SIBO, systemic inflammation and NAFLD [67], hence suggesting the importance of increased gut permeability. The severity of NAFLD is thus linked with SIBO and dysbiosis of gut microbiota. Based on these encouraging outcomes, studies are necessary to establish an exact cause and effective association between gut dysbiosis and NAFLD [2]. These results give credibility to hypothesize that improvement of the gut barrier disruption could help to alleviate the progression of the disease. Figure 1, highlights the significant role of SIBO in gut microbiota dysbiosis as well as the development of NAFLD $[13,17,25,35,55,61]$. Briefly, unique probiotic treatment approaches should be implemented, which can regulate the small intestinal bacterial overgrowth in the gut and thus could reduce the gut dysbiosis and this will ultimately mitigate the disease.

\section{Mechanisms in Dysbiotic Gut Microbiota: Impact on NAFLD?}

Dysbiotic gut microbiota and its several metabolites and endotoxins might be the characteristic of NAFLD progression through numerous mechanisms. Following are some major mechanisms showing a significant role in the progression and development of NAFLD.

\section{Crosstalk between Dysbiotic gut Microbiota and its Metabolites}

\section{Bile acids (BAs)}

Bile acids are amphipathic in nature, produced in the liver from cholesterol [68]. These are secreted in the biliary tract and reach the small intestine via the duodenum, combine with other components of the biliary tract, enable the emulsification, digestion, absorption of dietetic fats, cholesterol as well as emulsify fat-soluble vitamins [52]. Bile acids (BAs) are known as a significant regulator of lipid metabolism, glucose and energy homeostasis. Regulatory properties of bile acids have been well studied concerning the farnesoid $\mathrm{X}$ receptor (FXR), identified as a transcription factor (NR1H4) and Takeda G protein-coupled membrane receptor 5 (TGR5). These bile acid receptors regulate its endogenous synthesis and release as well as regulate several host metabolic pathways. It controls more metabolic functions via alterations in its transcriptional gene expression [69, 70]. It is showed that almost $95 \%$ of bile acids keenly reabsorb in the last part of ileum then transfer back to the liver [71]. While residual 5\% BAs deconjugated, dehydroxylated and dehydrogenated by colonic microbiota to procedure secondary bile acids (deoxycholic acid, lithocholic acid and ursodeoxycholic acid), reached the liver then to portal circulation through passive absorption [70,72].

The deconjugated BAs are less effective in formation of micelle and emulsification of ingested lipids parallel to conjugated BAs, so reduce its proficiency for fats absorption [73]. The connection between gut microbiota and NAFLD, which is 
moreover dependent on altered BAs metabolism via the gut microbiota [74]. Besides, gut microbiota could affect the homeostasis of BAs pool through deconjugating, also metabolizing primary BAs to secondary bile acids in gut, these toxic molecules take part in the modulation of lipids as well as energy metabolic pathways (Figure 2), and altered bile acid signaling receptors (FXR/TGR5), that can cause NAFLD [75-77]. However, little is known, which gut microbe is involved in this conversion. In addition, studies indicated that bile acids and gut microbiota are closely related and maintain each other. BAs directly regulates microbiota via binding to the FXR receptor, prompt the antimicrobial peptides (angiogenin1) and peptides of (RNase family 4), which directly inhibit the overgrowth of gut microbiota and subsequently mucosal barrier dysfunction [78].

Gut microbiota dysbiosis alters the balance among primary bile acids as well as secondary bile acids and their successive enterohepatic cycling, which metabolic properties are not precisely clear. An imbalance in bile acids and intestinal microbiota cause a cascade of immune reactions, inflammation and development of liver diseases [52]. Authors in a study have shown that NASH patients have altered bile acids composition with a preponderance of secondary bile acids [76, 79]. It is also revealed that the altered BAs destruct the cellular membranes via interaction with the membrane phospholipids, which results in bactericidal action [8]. Definitely, BAs are specific molecules for a G-protein-coupled receptor, similarly activates FXR (NR1H4), which downstream targets and shows a key role in regulation of hepatic de novo lipogenesis, plasma triglyceride turnover also very-low-density lipoprotein-triglyceride distribution [80]. TGR5 hinder secondary bile acids, stimulates glucose homeostasis via inducing the secretion of glucagon-like peptide 1 [69]. In a study supplementation of definite TGR5 agonists reduces triglyceride profile in serum as well as in the liver, thereby decreasing liver steatosis [81]. Maintaining the bile acid metabolism and microbiota can therefore, halt the progression of NAFLD [82]. Moreover, an association between gut microbiota and bile acids offers an important indication for the gut microbiota targeted treatment for NAFLD [76,83]. Thus, novel bile acids (BAs) regulatory probiotic strains are essential which may ameliorate the gut microbiota by regulating BAs pool and FXR activation.

\section{Choline}

Choline is an essential phospholipid and part of the cell membrane, shows a key role in lipid metabolism in the liver, in the assembly of very-low-lipoprotein. It encourages the transport of lipid from the liver [84]. Choline halts abnormal lipids accumulation in the liver, whereas deficiency of choline produces abnormal phospholipid, defective very low-density lipoproteins and causes alteration in the bile acids circulation (Figure 2), which commonly leads to hepatic steatosis. Several other factors also affect the bioavailability of choline, including food intake, estrogen status, single-nucleotide polymorphisms (SNPs) variation in genes for the de novo choline metabolism [85]. Nevertheless, gut microbiota metabolized the dietetic choline into a variety of toxic metabolic products, for example, trimethylamine and therefore can decrease the choline bioavailability [86].

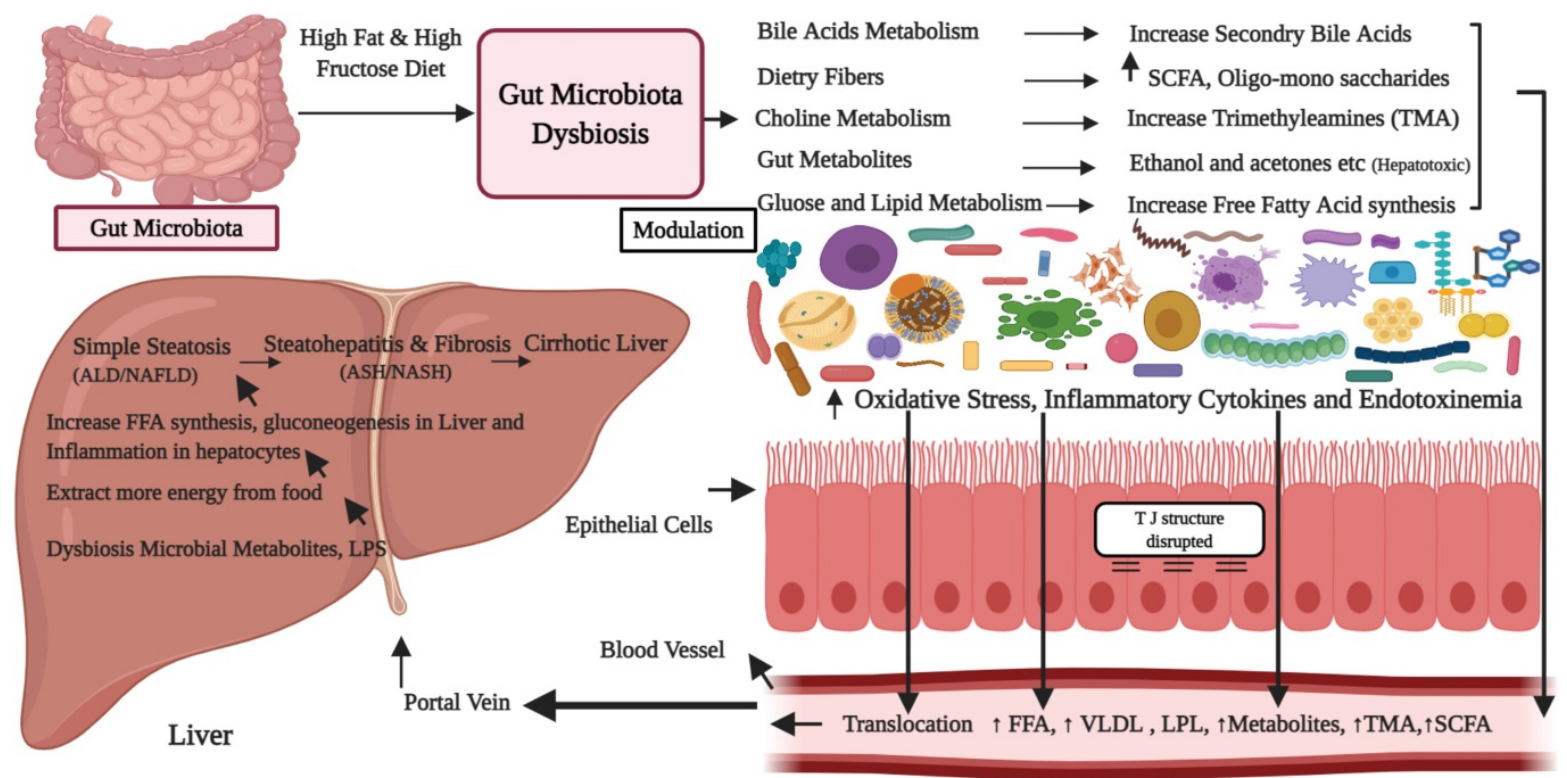

Figure 2. Proposed mechanisms showing the role of high-fat and sugar diets in the progression of gut microbiota dysbiosis and development of the nonalcoholic fatty liver disease. 
Gut microbiota produce enzymes (e.g. choline-TMA lyase, glycine betaine reductase), which catalyze the dietetic choline in toxic methylamines (dimethylamine and trimethylamine). Liver uptake these amines and convert into trimethylamine-Noxide, could produce liver inflammation as well as liver damage $[87,88]$. Evidence showed that choline-deficient food stimulates liver steatosis, reversible through choline infusion [89]. Hence, gut microbiota dysbiosis could stimulate NASH by reducing dietary choline levels, as well as raising toxic methylamines [85]. Studies described that feeding mice deficient with choline for 4 weeks induct NASH like disorder and produced many clinical signs and serum markers, high concentration of liver enzymes, hepatic triglycerides, lipid peroxidation as well as weight loss in mice [52, 90]. However, experiments conducted in germ-free mice indicate that mice do not produce such toxic methylamines, which support the important role of intestinal microbiota in the transformation of dietary choline to its intermediate compounds [91].

Normally, choline is converted into the phosphatidylcholine, for example, a lecithin compound in the host, which causes excretion of low-density lipoproteins particles from the liver and prevents hepatic triglycerides accumulation in the liver (steatosis) [92]. However, choline is also transformed to trimethylamine by gut microbiota, where trimethylamine can transfer to the liver and further change to trimethylamine $\mathrm{N}$-oxide [93]. Circulation of trimethylamine is implicated with a low concentration of host-derived phosphatidylcholine; an imbalance that commonly causes gut dysbiosis. Increased trimethylamine concentration has been related to liver injury due to a higher accumulation of triglycerides in the liver [94]. Increased triglycerides (hepatic steatosis) results in NAFLD in humans as well as in experimental animal models [95]. Further, NAFLD patients are associated with SIBO and its demand for phosphatidylcholine increases, for its growth as well as division; this might contribute to the deficiency of choline in the host and subsequently contribute to NAFLD as well as NASH [2]. Accordingly, a close and benefited relationship exists among gut microbiota and choline metabolism, which provides a significant role of gut microbiota-targeted treatment for NAFLD [83]. Thus, modifying the metabolic profiles of gut microbiota may serve as unique microbiome-based strategies for the management of NAFLD [96].

\section{Ethanol and acetaldehyde}

Gut microbiota produces several potential hepatotoxic molecules, for example, ethanol, acetaldehyde, ammonia and phenols, which are transported to the liver via portal circulation system. The gut mucosa directly absorbs maximum ethanol from food, beverages, stomach as well as small intestine via simple diffusion, while the biggest part of the alcohol (ethanol) produced by microbial fermentation comes from large intestine through the systemic circulation [11]. Interestingly, studies showed that NAFLD is progressed by increased circulating as well as luminal levels of ethanol, its metabolites, like acetaldehyde and acetate $[11,96]$. The gut microbiota plays an important role in pathogenesis by numbers of alcohol-producing bacteria largely Escherichia coli, which has been established to raise gut epithelial permeability. In addition, the elevated level of serum ethanol (Figure 2), play a major role in oxidative stress, liver inflammation and development of NASH [39]. Besides E. coli, other bacterial genera, like Bacteroides, Bifidobacterium, as well as Clostridium, are also shown to produce alcohol, causes important ethanol mediated injury [11]. Ethanol is most common as well as frequent metabolite of various hetero-lactic organisms and endogenously gut microbes produced it. It is responsible for physiological and morphological differences, especially causes activation of macrophages, pro-inflammatory cytokines production, through which gut bacteria might interrupt the intestinal barrier, leads to small intestinal bacterial overgrowth and therefore enhances translocation of endotoxins into the portal circulation [97]. Further, apart from the disruption of the gut barrier integrity, it is also associated with the secretion of pro-inflammatory liver cytokines and pathogenesis of NAFLD [98]. However, the precise mechanisms of the present hypothesis need more study [28].

Likewise, acetaldehyde and its metabolites also stimulate the Kupffer cells, which activate the innate immune system, increase the production of nitric oxide, cytokines, which causes the production of ROS [99]. Acetaldehyde is involved in weakening of the gut tight junction proteins, compromises gut barrier integrity and allowing translocation of the microbial products [100]. It is also linked with down-regulation of the expression of AMPs in the gut [101], causes its permeability, which leads to disruption of gut barrier functions, proliferates the oxidative stress and ultimately induces the liver injury [102]. The liver reacts to circulating ethanol by up-regulating its ethanol metabolic pathway [103]. Gut microbiota as well as enterocytes produce alcohol metabolizing enzymes (alcohol dehydrogenase), that cometabolizes alcohol (ethanol) into major metabolites like acetaldehyde as well as acetate [104]. This shows 
that modulation of gut microbiota produces various altered metabolites, which ultimately leads to liver inflammation and the onset of NAFLD.

\section{Short-chain fatty acids}

Short-chain fatty acids (SCFAs), like acetic acids, propionic acids and butyric acids are commonly produced by fermentation of gut microbes [105]. The gut microbes supply substrate for fermentation of the complex indigestible dietary polysaccharides to make SCFAs and support the host in getting maximal energy [39]. These SCFAs are rapidly absorbed in the colon and have many functions in maintaining the inflammation, gut motility, glucose, lipid homeostasis and energy harvesting [106]. However, overproduction of the SCFAs might promote lipogenesis in the liver as they use it as a substrate for lipogenesis [107].

The various gut metabolites produce mutable effects on the host and some of them could be beneficial for synthesis of signaling molecules like butyrate [39], that not only supply energy for gut mucosal cells but also prevent the production of cytokines and reduce the incidence of chronic metabolic inflammation via GPR43 pathway [108]. Qin et al. have revealed that the composition of gut microbiota in the cirrhosis patients is characterized by the lower number of butyrate synthesizing bacteria as compared to healthy subjects, which have anti-inflammatory characteristics [109]. Butyric acid activates the peptide AMP-activated protein kinase, in the liver [110], therefore, improve the gut barrier dysfunction [111]. Similarly, in a study it was shown that butyrate could efficiently improve the lipid accumulation as well as liver inflammation in animal models of NAFLD via modulation of gut microbiota and barrier functions [112], reduce the induction of induced nitric oxide synthase [113], and suppress the inflammatory pathways [114].

In the gut dysbiosis state, bacterial species of Bacteroidetes are identified as the main contributor to acetate and propionate production, whereas Firmicutes as a key producer of butyrate. Increased level of propionic acid is shown to promote gluconeogenesis in the liver [115]. SCFAs triggered the expression of $G$ protein-coupled receptors (GPRs), especially the GPR41 and GPR43, which could encourage the secretion of the peptides GLP1 [116], and stimulate the genes in the liver cells that regulate the fatty acid $\beta$-oxidation pathway and also insulin sensitivity [117], thus promoting the occurrence as well as progression of NAFLD (Figure 2). Experiments have determined that feces of obese individuals have high levels of SCFAs then lean individuals, suggesting that gut microbiota from the obese individuals exhibit the increased capability to extract and store energy from food as compared to lean ones [118]. The role of other short-chain fatty acids, for example, acetic acid as well as propionic acid, could directly infect the hepatocytes, adipose tissue, as well as central nervous system [119], which needs further study. Studies are required to validate how SCFAs can play a role in the pathogenesis of nonalcoholic fatty liver disease/NASH subjects [2]. Figure 2, highlights the proposed mechanisms showing the role of high-fat and sugar diets in the progression of gut microbiota dysbiosis and development of the nonalcoholic fatty liver disease [2, $51,52,70,92,97,107]$. In fact, high-fat diets and high sugar results in gut dysbiosis and small intestinal bacterial overgrowth (SIBO). Alterations in gut microbiota (dysbiosis) increase energy extraction from the gut and alter the normal dietetic choline, BAs, lipids and glucose metabolism. This consequently increases the production of endotoxins, pro-inflammatory cytokines, increase the gut epithelial permeability and toxins affect the gut liver axis, which finally results in NAFLD/NASH. In summary, more studies are essential to determine the significant characteristics of SCFAs and especially its role in the NAFLD and it is essential to completely understand the thorough molecular mechanisms and consequences of these hepatotoxic compounds on the liver.

\section{How Probiotics Role as Therapeutic Strategy for Management of NAFLD?}

The gut microbiota plays a vital role in humans through supporting the gut homeostasis, protection against pathogens, enhancing the development of the immune system, also supporting in production of energy as well as micro-nutrients [120]. It could be easily presumed that gut microbiota shows a fundamental role in maintaining human health [106]. Indeed, changes in the composition of microbiota have been linked to several metabolic syndromes including NAFLD $[10,121,122]$. It is hypothesized that gut microbiome restoration might be a better method for treatment of NAFLD [123-125]. Probiotics have been proposed to restore the dysbiosis gut to the healthy gut microbiome and administration of probiotics can be a treatment approach for prevention and control of chronic liver diseases because they are non-pathogenic $[25,26,50]$, and can prevent translocation of harmful bacteria and endotoxins by inhibiting their epithelial invasion, preventing bacteria adherence to mucosal surface, decreasing inflammatory responses via the production of antimicrobial peptides and furthermore stimulating the host immunity [50]. Evidence showed that 
probiotics boost intestinal microbiota to re-establish intestinal-mucosal cross-talk which reduces the progression of NALFD [126].

A significant number of studies revealed that probiotics normalize the gut epithelial homeostasis, i.e. promote cell persistence and gut barrier functions by preventing the translocation of LPS thus alleviate eubiosis, improve gut bacterial ecology as well as adjust the immune functions $[49,127]$. Continuing experimental trials involving probiotics in NAFLD [128], could bring more development in this research field from the basic research towards the clinical practice. Recently encouraging outcomes of probiotics are shown in pre-clinical studies in children as well as adults with NAFLD. Nevertheless, randomized controlled investigations are limited in these populations [24,129]. Therefore, large scale clinical studies are essential to understand the efficacy of probiotics against NAFLD.

\section{Probiotics}

According to FAO/WHO, Probiotics can be defined, as "live, non-pathogenic microbes when administered in the appropriate quantities, confer a benefit to the host health" [22]. Probiotics are traditionally fermented foods and the Lactobacilli as well as Bifidobacteria, are the main bacteriological groups used as probiotics [130, 131]. Conversely, for most of the commercially available probiotic strains the parameters such as quality control, including its characterization, viability, formulation and safety issues are rarely addressed [25]. Generally, the candidate probiotic strains that to be used for treatment should strictly follow the proposed WHO guidelines before commercialization in the market [132].

The ideal candidate probiotic strain (n) must have the features to take part in indigenous colonization [133], originated from the human, non-pathogenic [134]. It should hinder the growth of various pathogens, survive in the human optimum body temperature, immune to antibiotic therapy. It lives throughout the gastrointestinal tract, oppose to bile salts (pancreatic juice) [132, 133]. Probiotic strains should live in duodenum environment, stimulate the immune system via the production of the antagonistic proteins (bacteriocins) to pathogens, produce short-chain fatty acids to improve the epithelial barrier and to increase the anti-inflammatory action and balance the gut microbiota [132]. Additionally, the candidate probiotics should have a protecting role in the host against the oxidative stress, anti-cholesterol, anti-obesity, and anti-diabetes $[132,135]$.
Studies illustrate that the probiotics become the source of modulation and functions of endogenous microbiota and disturbing its interaction with the host, also causes the competitive elimination of pathogens. Further, available fact shows that probiotics can inhibit obesity as well as NAFLD by improving fatty acid oxidation, promote the mucosal barrier function, mucosal recovery and regulate innate immune responses in pathological situations [23]. Therefore, manipulating the gut microbiota can decrease the endotoxins and other hepatotoxic toxic compounds and further effective in reducing the inflammation, increase the fatty acid oxidation [134].

Recently, an investigation has shown that probiotics have the tendency to alter the dysbiosis gut microbiome towards the beneficial [24]. Figure 3, illustrates the probiotics mechanisms of action in alleviating the host gut microbiota homeostasis [23, $25,26,50,120,132,137]$.

Overall, the beneficial effects of different probiotic strains on NAFLD have shown. However, it is not fully elucidated about specific probiotics, that either individually they are more effective or in combination, for the treatment of NAFLD. Therefore, future studies are needed to discover a unique and specifically effective probiotic (n) that fully attenuates or treats the key source of this disease, which will help to enhance the therapeutic potential of NAFLD.

\section{Therapeutic role of probiotics on NAFLD in animal model studies}

Several animal model studies have shown evidence that probiotics can reduce the progression of NAFLD [134,138]. They could recover the gut microbiota configuration, liver pathology via the down-regulation of serum LPS and liver TLR4. It is proposed that both alteration of gut microbiota and their endotoxemia could be involved in the development of NAFLD and probiotics can delay the development of NAFLD by restoring the gut microflora, improve the expression of tight junction proteins (occludin), which can inhibit endotoxin circulation in blood by the LPS/TLR4 signaling [139]. Probiotic therapy was conducted and shown that Lactobacillus plantarum could alleviate the NAFLD by maintaining fat metabolism such as activating the AMPK pathway into phosphorylate ACC and further halt SREBP-1/FAS signaling, which may inhibit de novo lipogenesis as well as increase the fatty acid oxidation. Furthermore, treatment with L. plantarum NA136, Nrf-2 (transcription factor) was increased which might activate the antioxidant pathway, that could decline in pro-inflammatory cytokines and thus protect against the oxidative stress [134]. Other animal model studies on NAFLD, alcoholic liver disease 
(ALD), along hepatic encephalopathy (HE) have described the valuable properties of certain probiotic strains on liver damage. Especially, in experimental studies of NAFLD, the importance of probiotic strains have been shown [140]. It was noted that oral supplementation of L. plantarum strains in mice with HFD, can ameliorate the progression of NAFLD by regulating the liver functions, fecal microbiota, improves the expression and activities of antioxidant enzymes and downregulated the lipogenesis associated genes [141]. Similarly, supplement with a mixture of probiotic preparation modulated the gut epithelial permeability i.e. maintain the tight-junction proteins (ZO-1 and $\mathrm{ZO}-2$ ), attenuated inflammation and also reduced the concentration of liver triglyceride [142]. Pretreatment of the individual mice with oral probiotics prevented the disruption in gut barrier function (zonula occludens-1), significantly reduced the liver damage and decreased the bacterial translocation by improving the gut mucin expression (MUC2), and inhibiting against LPS or TNF-a induced gut barrier injury [143]. In a latest study, authors isolated novel probiotic strain (Bifidobacterium animalis subsp. lactic Bb12), from the healthy Mongolian youngsters and orally administration of $B$. animalis subsp. lactic V9, significantly mitigated the
HFD-induced increases in ALT, AST, ameliorated hepatic steatosis, reduced the transcription of PPAR-a, SREBP-1c, FAS. Further, V9 administration restored the expression of hepatic phosphorylatedAMPK and suppressed the inflammatory cytokines like, IL-6, IL-1 $\beta$, TNF- $\alpha$ in the HFD fed rats. The anti-inflammatory properties of $B$. animalis subsp. lactic was shown to be connected with suppression of hepatic gene expression (e.g. TLR4, TLR9 and NLRP3 and ASC mRNA), in the HFD rats. These encouraging results shows that $\mathrm{V} 9$ alleviate NAFLD via regulating de novo lipid synthesis, suppressing the inflammation by AMPK as well as TLR-NF-kB pathways [136]. More excitingly, it was showed that probiotic mixture can promise to treat the pathogenesis of NAFLD and could mitigate the high fat/high sugar diet-induced obesity via increasing the gut beneficial bacteria and reducing the pro-inflammatory gut bacteria [144].

In addition, researchers identified that multi-strain probiotics can prove more valuable than single probiotic against fat accumulation and microbiota modulation in diet-induced obesity [125, 145]. In a study, a mixture of Bacillus animalis VKB/ Bacillus animalis VKL/Lactobacillus casei strains, revealed more effective than a strain Lactobacillus casi in reducing the weight of the obese mice, cholesterol

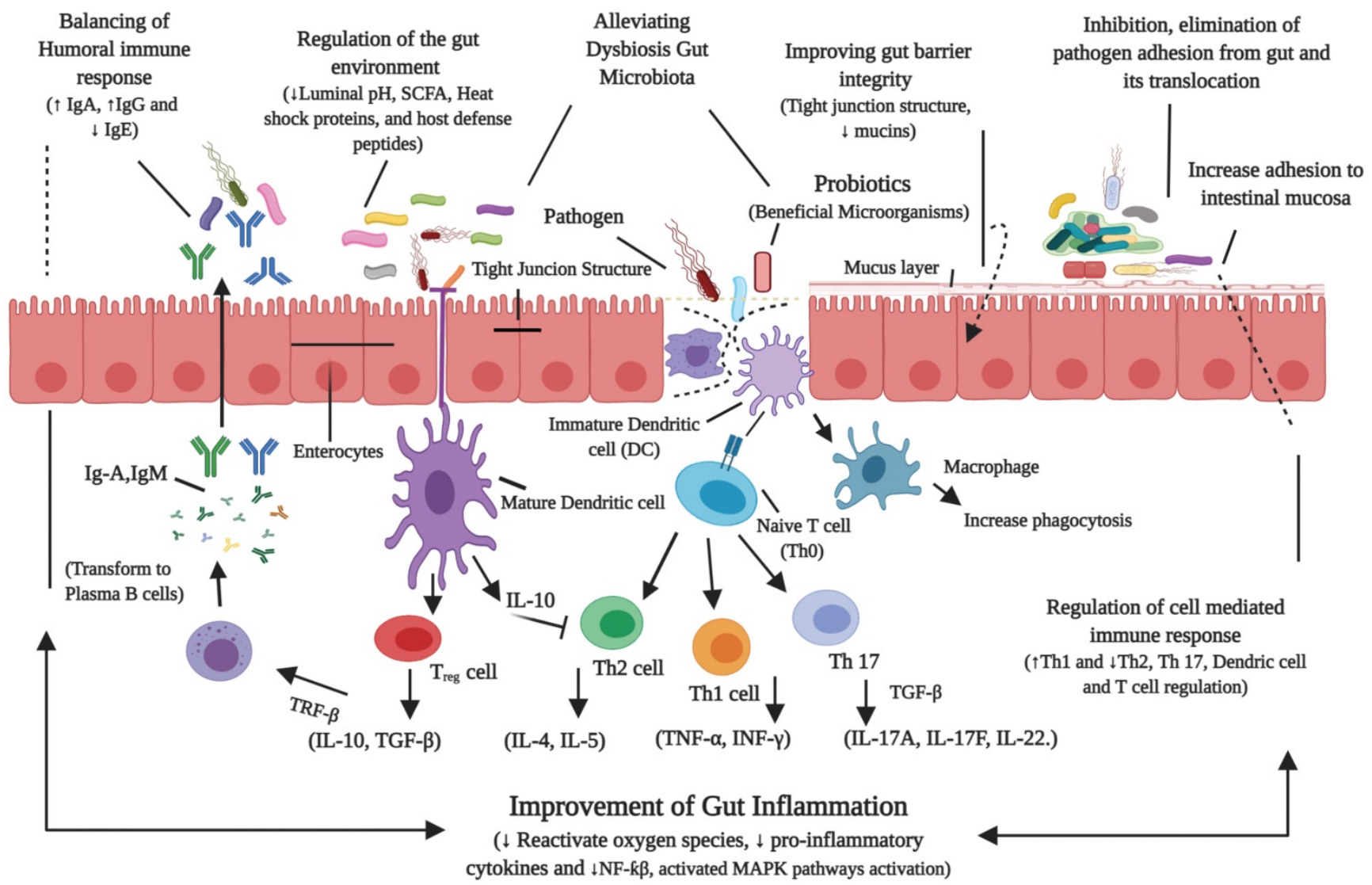

Figure 3. Probiotics mechanisms of action in alleviating the host gut microbiota homeostasis. Probiotics could alleviate the gut disorders (NAFLD), by modulating the gut microbiota, prevent the translocation of bacterial toxins, reduce the gut permeability and regulate the immune response. 
level, modulating gut microbiota in energy rich diet and further restoring the morphology of liver [146]. In another recent study, probiotics mixture was supplemented in combination, which significantly alleviated the gut microbiota, reduced the fats in liver, alleviated aminotransferase activity and improved the level of TNFa, IL-6, IL-8 in HFD-induced NAFLD rats. It also decreased the expression of HMG-CR and up regulate the PPAR-a levels whereas down regulate the PPAR- $\gamma$ as well as SREBP-1C levels [147]. Similarly, it has been found that a mixture of probiotics can alleviate NAFLD by improving the energy, fat metabolism and regulating the gut microbiota [148]. However, further research is necessary to explore specific probiotic strains and understand its role in combination for the management of gut microbiome in NAFLD patients. Table 2, highlights the role of probiotic strain (n) in experimental studies to treat NAFLD.

\section{Therapeutic role of probiotics on NAFLD in human studies}

Experimental studies on NAFLD showed the importance of probiotics. However, less data is available on humans as compared to animal studies. Preliminary data revealed considerably the effectiveness of several probiotics and compared them with control in NAFLD patients [59]. In addition, two double-blind and placebo-controlled studies, shown an important decrease in liver enzymes (ALT, AST), triglycerides, LDL-C, with supplementation of probiotics in obese children [149]. Malaguarnera et al. found that oral supplementation of Bifidobacterium longum along with the fructo-oligosaccharides and modification of lifestyle, improved alanine aminotransferase level (ALT), cholesterol, TNF-a, lipoprotein, endotoxins in serum profile, improve the insulin resistance (IR) and hepatic steatosis index in NASH patients [150]. These encouraging results intensely indicate the benefits of probiotics in the medication of NAFLD. However, as shown in a meta-analysis, still, more studies are required about the usage of probiotics in treatment of NAFLD [151].

Table 2. Role of probiotic strains in experimental studies to treat NAFLD

\begin{tabular}{|c|c|c|c|c|}
\hline Probiotic strain(n) & Studies/Model & $\begin{array}{l}\text { Study } \\
\text { Duration }\end{array}$ & Outcome after treatment/Main Effects & $\begin{array}{l}\text { Year-Author } \\
\text { [Ref:] }\end{array}$ \\
\hline $\begin{array}{l}\text { Lactobacillus reuteri } \\
\text { GMNL-263 }\end{array}$ & $\begin{array}{l}\text { High fructose diet (HFD) } \\
\text { fed rats }\end{array}$ & 14 weeks & $\begin{array}{l}\text { Improve the gut microbiota, antioxidant enzymes, IL-6, TNF-a levels, PPAR- } \gamma \text {, } \\
\text { GLUT4 mRNA expression level and overall } \\
\text { improving hepatic steatosis }\end{array}$ & $\begin{array}{l}\text { Hsieh }-2013 \\
{[145]}\end{array}$ \\
\hline $\begin{array}{l}\text { Lactobacillus rhamnosus } \\
\text { GG }\end{array}$ & $\begin{array}{l}\text { Mice: High fructose diet } \\
\text { induced NAFLD }\end{array}$ & 8 weeks & $\begin{array}{l}\text { Keep mice from NAFLD, improve gut beneficial bacteria, liver enzymes, } \\
\text { diminished liver inflammation as well as steatosis }\end{array}$ & $\begin{array}{l}\text { Yvonne-2014 } \\
{[140]}\end{array}$ \\
\hline $\begin{array}{l}\text { Lactobacillus Rhamnosus } \\
\text { LGG, Lactobacillus } \\
\text { plantarum WCFS1 }\end{array}$ & $\begin{array}{l}\text { Rats: High fat diet } \\
\text { induced NAFLD }\end{array}$ & 21 weeks & $\begin{array}{l}\text { Ameliorate the intestinal microbiota, gut barrier structures, level of gut } \\
\text { endotoxemia, inflammation, lipid metabolism and IR via enhancing the } \\
\text { expression levels of CYP7A1 and LDL-R in liver }\end{array}$ & $\begin{array}{l}\mathrm{Lu}-2015 \\
{[147]}\end{array}$ \\
\hline Probiotic mixture & $\begin{array}{l}\text { Mice: HSHF diet induced } \\
\text { NAFLD }\end{array}$ & 6 weeks & $\begin{array}{l}\text { Alleviate the body mass, blood } \\
\text { triglyceride level, total cholesterol level, highest anti-obesity, } \\
\text { antioxidant properties }\end{array}$ & $\begin{array}{l}\text { Song }-2016 \\
{[135]}\end{array}$ \\
\hline Probiotic mixture & $\begin{array}{l}\text { Rats: HFD-induced } \\
\text { NAFLD }\end{array}$ & 12 weeks & $\begin{array}{l}\text { Improve the gut microbiota, delay the development of NAFLD } \\
\text { through LPS/TLR } 4 \text { signaling } \\
\text { pathway, improve hepatic fats accumulation and liver enzymes }\end{array}$ & $\begin{array}{l}\text { Xue }-2017 \\
{[139]}\end{array}$ \\
\hline Probiotic mixture & $\begin{array}{l}\text { Rats: High fat diet } \\
\text { induced NAFLD }\end{array}$ & 5 weeks & $\begin{array}{l}\text { Enhance the gut microbiota composition, liver functions, cholesterol level, } \\
\text { oxidative stress and lipid metabolism }\end{array}$ & $\begin{array}{l}\text { Bubnov - } 2017 \\
{[146]}\end{array}$ \\
\hline Probiotic mixture & HFD-induced obese mice & 12 weeks & $\begin{array}{l}\text { Improve the gut microbiota, up-regulate fatty acid oxidation related genes } \\
\text { (PPARa, AOX), in liver and adipocytes, alleviated IL-6 levels }\end{array}$ & $\begin{array}{l}\text { Kim }-2017 \\
{[138]}\end{array}$ \\
\hline $\begin{array}{l}\text { Lactobacillus plantarum } \\
\text { NA136 }\end{array}$ & $\begin{array}{l}\text { HFD/ fructose diet } \\
\text { induced NAFLD mice }\end{array}$ & 16 weeks & $\begin{array}{l}\text { Ameliorate NAFLD, prevent de novo lipogenesis, increase fatty acid oxidation. } \\
\text { Reduced weight gain, decreased lipids, mass of tissues fat, serum ALT, AST } \\
\text { levels }\end{array}$ & $\begin{array}{l}\text { Zhao-2019 } \\
{[134]}\end{array}$ \\
\hline $\begin{array}{l}\text { L. acidophilus, } \\
\text { Bifidobacterium longum, } \\
\text { Enterococcus faecalis }\end{array}$ & $\begin{array}{l}\text { C57BL/6J mice: HFD fed } \\
\text { mice }\end{array}$ & 4 weeks & $\begin{array}{l}\text { Probiotics mitigate the diet-induced obesity, restored the beneficial gut } \\
\text { bacteria, reducing serum total cholesterol level, triglycerides and LDL } \\
\text { cholesterol }\end{array}$ & $\begin{array}{l}\text { Kong-2019 } \\
{[144]}\end{array}$ \\
\hline $\begin{array}{l}\text { Lactobacillus plantarum } \\
\text { mixture }\end{array}$ & $\begin{array}{l}\text { Wistar rats: High fat and } \\
\text { sucrose diet induced } \\
\text { nonalcoholic fatty liver } \\
\text { disease }\end{array}$ & 8 weeks & $\begin{array}{l}\text { Mitigate the pathogenesis and steatosis in NAFLD through regulating liver } \\
\text { function and gut microbiota, downregulated de novo lipogenesis associated } \\
\text { genes and increased antioxidant enzymes }\end{array}$ & $\begin{array}{l}\text { Park - } 2020 \\
{[141]}\end{array}$ \\
\hline $\begin{array}{l}\text { Bifidobacterium animalis } \\
\text { subsp. lactis V9 }\end{array}$ & $\begin{array}{l}\text { Wistar rats: high-fat diets } \\
\text { induced NAFLD }\end{array}$ & 9 weeks & $\begin{array}{l}\text { Regulate the liver function enzymes, ameliorated hepatic steatosis, suppressed } \\
\text { inflammatory cytokines, modulate accumulation of the hepatic triglyceride, } \\
\text { free fatty acid, increase glycogen level. Decreased serum glucose level, restored } \\
\text { the expression of hepatic phosphorylated-AMPK, and reduced the } \\
\text { transcription of PPAR-a, SREBP-1c, FAS }\end{array}$ & $\begin{array}{l}\text { Yan }-2020 \\
{[136]}\end{array}$ \\
\hline
\end{tabular}


Table 3. Role of probiotic strains in clinical studies to treat NAFLD patients

\begin{tabular}{|c|c|c|c|c|}
\hline Probiotic strain(n) & $\begin{array}{l}\text { Studies/Model/ } \\
\text { patients }\end{array}$ & $\begin{array}{l}\text { Study } \\
\text { Duration }\end{array}$ & Outcome after treatment/Main Effects & Year-Author [Ref:] \\
\hline Lactobacillus rhamanus & 20 Obese children & 8 weeks & $\begin{array}{l}\text { Reduce the body weight, alleviate ALT, AST and } \\
\text { tumor necrosis factor-alpha levels }\end{array}$ & Vajro-2011 [157] \\
\hline $\begin{array}{l}\text { Bifidobacterium longum+ } \\
\text { Fructo-oligosaccharides (FOS) }\end{array}$ & $\begin{array}{l}\text { Randomized, double blind } \\
\text { and placebo controlled trial, } \\
66 \text { NAFLD (biopsy proven) } \\
\text { patients }\end{array}$ & 24 weeks & $\begin{array}{l}\text { Improve TNF-a, C-reactive protein, AST, } \\
\text { endotoxin levels, HOMA-IR, liver steatosis and } \\
\text { NASH }\end{array}$ & Malaguarnera - 2012 [150] \\
\hline $\begin{array}{l}\text { Lactobacillus acidophilus (La5), } \\
\text { Bifidobacterium lactis (Bb12) }\end{array}$ & $\begin{array}{l}\text { Double-blind, randomized } \\
\text { and controlled trial, } 72 \\
\text { patients with NAFLD }\end{array}$ & 8 weeks & $\begin{array}{l}\text { Alleviate AST, alanine aminotransferase, serum } \\
\text { triglycerides (TC), total cholesterol and LDL } \\
\text { cholesterol }\end{array}$ & Nabavi-2014 [158] \\
\hline $\begin{array}{l}\text { Lactobacillus Acidophilus, } \\
\text { Bifidobacterium bifidum, Bifidobacterium } \\
\text { lactis and Lactobacillus rhamanus }\end{array}$ & $\begin{array}{l}\text { Randomized, triple blind } \\
\text { trial, } 64 \text { children with } \\
\text { obesity-related liver } \\
\text { syndromes(NAFLD) }\end{array}$ & 12 weeks & $\begin{array}{l}\text { Enhance liver function enzymes level, reduced } \\
\text { lipopolysaccharide levels, triglycerides }\end{array}$ & Famouri-2017 [149] \\
\hline $\begin{array}{l}\text { Probiotic mixture (Bifidobacterium } \\
\text { Lactobacillus \& Enterococcus, B. subtilis } \\
\text { and Enterococcus) }\end{array}$ & 200 NAFLD patients & 1 month & $\begin{array}{l}\text { Ameliorate the gut microecological structure in } \\
\text { NAFLD patients, decreased serum ALT, AST } \\
\text { levels, inhibiting TNF-a level and enhancing } \\
\text { adiponectin level. Improved fatty liver, blood } \\
\text { lipids and blood glucose level, lipid metabolism } \\
\text { and protect liver injury }\end{array}$ & Wang - 2018 [57] \\
\hline Bifidobacterium animalis & 34 NAFLD patients & 24 weeks & $\begin{array}{l}\text { Alleviate hepatic steatosis, serum ALT, aspartate } \\
\text { aminotransferase level }\end{array}$ & $\begin{array}{l}\text { Bakhshimoghaddam }-2018 \\
\text { [156] }\end{array}$ \\
\hline Probiotic mixture & 68 obese NAFLD patients & 12 weeks & $\begin{array}{l}\text { Reduce body weight, total body fat, decreased } \\
\text { triglyceride, ALT, AST levels }\end{array}$ & Ahn - 2019 [159] \\
\hline
\end{tabular}

TNF-a: tumor necrosis factor alpha; HOMA-IR: homeostatic model assessment of insulin resistance; IL-6: interleukin 6; IL-10: interleukin 10; LDH: lactate dehydrogenase; CRP: c-reactive protein; IR: insulin resistance; HDL-cholesterol: high-density lipoproteins-cholesterol; LDL-cholesterol: low-density lipoprotein cholesterol; TG: triglycerides.

Study showed that the maintenance of gut flora might be a novel therapeutic approach in regulating of NAFLD and probiotics can modulate the gut microbiota [26]. Further, a meta-analysis confirmed that probiotic treatment effectively reduced the liver enzyme profile, however, their effects on the improvement of lipid profile were not significant [152]. Further, some studies did not show the supportive effects of probiotics and have shown conflicting clinical findings for several probiotic strains and their formulations [153], particularly in treatment of gut barrier dysfunctions, as indicated in patients with abdominal surgery. Altogether following side effects (i.e. usually noted BT, gut colonization with enteric bacteria also septic morbidity), after surgery were not recovered using an oral dose of Lactobacillus plantarum 299v for one week [154]. The rules for studying liver diseases given by American Gastroenterological association do not encourage probiotics for the treatment of NAFLD [155]. This indicates that probiotics must be safe and effective when consuming for management of the NAFLD. However, current research might give more support to it in the future. Although, too much literature is available on the subject area and undoubtedly the animal models on NAFLD and numerous probiotic strains have been studied in various studies. Though, it is tough to conclude about the accurate effect of probiotics on NAFLD patients. Therefore, detailed large-scale clinical studies are required in human volunteers with NAFLD to insure the benefits of novel probiotic strains/therapeutic approaches on dysbiosis gut microbiota and in the treatment of NAFLD. Table 3, highlights the role of probiotic strains in clinical studies to treat NAFLD patients.

\section{Conclusion and Prospects}

In summary, human gut microbiota maintains normal health and gut functions. Its alteration leads to pathogenic microbes and gut-derived products, which play a significant role in the progression of diseases in humans, such as NFALD, obesity, diabetes and other metabolic syndromes. Although NFALD is a multi-factorial liver disease and gut microbiota alteration (dysbiosis) is considered a novel factor to be involved in its pathogenesis, studies have shown the mechanism of this disease, but its other causality is yet to be established. There is a need to explain the exact mechanisms of gut liver axis interactions, gut dysbiosis and feasible therapeutic options. Gut microbiota restoration will be a milestone in the management of this disease. A better understanding of the individual gut microbiome, disease pathogenesis and the ideal probiotic strain(n) would be the best approach for the management of NAFLD. Encouraging results of most commonly used probiotics have shown on pathophysiological symptoms of the liver and many therapies are still under study. Still, there is no treatment for NAFLD. A number of studies about this disease come from animal models and most of the experimental studies have used a single strain of probiotics. A limited data on human studies are available, therefore a large scale 
of human studies using a combination of specific probiotics are required, which can strongly reduce or even eliminate the prime factors of this disease and can maintain the normal microbiota of the gut. In addition, large-scale, efficient randomized clinical trials (RCTs) might be needed particularly, using advanced tools like omics technologies, to elucidate the exact mechanism(s) of action and achievable therapeutic avenues for NAFLD.

\section{Abbreviations}

HFHFD: high fat and high fructose diet; SIBO: small intestine bacterial overgrowth; BT: bacterial translocation; TJ: tight junction; FFA: free fatty acid; IR: insulin resistance; ALD: alcoholic liver disease; NAFLD: nonalcoholic fatty liver disease; ASH: alcoholic steatohepatitis; NASH: nonalcoholic steatohepatitis; SCFA: short chain fatty acid; VLDL: very low-density-lipoproteins; LPL: lipoprotein lipase; LPS: lipopolysaccharide; Th: T helper cells; Treg: regulatory $\mathrm{T}$ cells; Ig: immunoglobulin; IL: interleukins; TNF: tumor necrosis factor; TGF: transforming growth factor; IFN: interferon; DC: dendritic cell; SCFA: short chain fatty acid; $M$ : macrophage; NF-Kb: nuclear factor-KB; MAPK: mitogen-activated protein kinase; ROS: reactive oxygen species.

\section{Acknowledgements}

The authors appreciate the numerous financial support by the Fundamental Research Funds for the Central Universities of China (Project number: lzujbky-2017-k15).

\section{Competing Interests}

The authors have declared that no competing interest exists.

\section{References}

1. Henao-Mejia J, Elinav E, Jin C, Hao L, Mehal WZ, Strowig T, et al. Inflammasome-mediated dysbiosis regulates progression of NAFLD and obesity. Nature. 2012;482:179-185.

2. Kolodziejczyk AA, Zheng D, Shibolet O, Elinav E. The role of the microbiome in NAFLD and NASH. EMBO Molecular Medicine. 2019;11:e9302.

3. Estes C, Razavi H, Loomba R, Younossi Z, Sanyal AJ. Modeling the epidemic of nonalcoholic fatty liver disease demonstrates an exponential increase in burden of disease. Hepatology. 2018;67:123-133.

4. Delzenne NM, Cani PD, Everard A, Neyrinck AM, Bindels LB. Gut microorganisms as promising targets for the management of type 2 diabetes. Diabetologia. 2015;58:2206-2217.

5. Cotter TG, Rinella M. NAFLD 2020: The State of the Disease. Gastroenterology. 2020;158:1851-1864

6. Gakidou E, Afshin A, Abajobir AA, Abate KH, Abbafati C, Abbas KM, et al. Global, regional, and national comparative risk assessment of 84 behavioural, environmental and occupational, and metabolic risks or clusters of risks, 1990-2016: A systematic analysis for the Global Burden of Disease Study 2016. The Lancet. 2017;390:1345-1422.

7. Pearson-Stuttard J, Zhou B, Kontis V, Bentham J, Gunter MJ, Ezzati M. Worldwide burden of cancer attributable to diabetes and high body-mass index: a comparative risk assessment. The Lancet Diabetes \& Endocrinology. 2018;6:95-104.
8. Ipsen DH, Lykkesfeldt J, Tveden-Nyborg P. Molecular mechanisms of hepatic lipid accumulation in non-alcoholic fatty liver disease. Cellular and Molecular Life Sciences. 2018;75:3313-3327.

9. Jensen VS, Tveden-Nyborg P, Zacho-Rasmussen C, Quaade ML, Ipsen DH, Hvid $\mathrm{H}$, et al. Variation in diagnostic NAFLD/NASH read-outs in paired liver samples from rodent models. Journal of Pharmacological and Toxicological Methods. 2020;101:106651.

10. Knight R, Callewaert C, Marotz C, Hyde ER, Debelius JW, McDonald D, et al. The Microbiome and Human Biology. Annual Review of Genomics and Human Genetics. 2017;15:397-411.

11. Zhu L, Baker SS, Gill C, Liu W, Alkhouri R, Baker RD, et al. Characterization of gut microbiomes in nonalcoholic steatohepatitis (NASH) patients: A connection between endogenous alcohol and NASH. Hepatology. 2013;57:601-609.

12. Caussy C, Soni M, Cui J, Bettencourt R, Schork $\mathrm{N}$, Chen $\mathrm{CH}$, et al. Nonalcoholic fatty liver disease with cirrhosis increases familial risk for advanced fibrosis. Journal of Clinical Investigation. 2017;127:2697-2704.

13. Sharpton SR, Ajmera V, Loomba R. Emerging role of the gut microbiome in nonalcoholic fatty liver disease: from composition to function. Clinical Gastroenterology and Hepatology. 2019;17:296-306.

14. Vallianou N, Stratigou T, Christodoulatos GS, Dalamaga M. Understanding the role of the gut microbiome and microbial metabolites in obesity and obesity-associated metabolic disorders: Current evidence and perspectives. Current Obesity Reports. 2019;8:317-332.

15. Jia W, Rajani C. The influence of gut microbial metabolism on the development and progression of non-alcoholic fatty liver disease. Advances in Experimental Medicine and Biology. 2018;1061:95-110.

16. Schwimmer JB, Johnson JS, Angeles JE, Behling C, Belt PH, Borecki I, et al. Microbiome Signatures Associated With Steatohepatitis and Moderate to Severe Fibrosis in Children With Nonalcoholic Fatty Liver Disease. Gastroenterology. 2019;157:1109-1122.

17. Amabebe E, Robert FO, Agbalalah T, Orubu ES. Microbial dysbiosis-induced obesity: role of gut microbiota in homoeostasis of energy metabolism. British Journal of Nutrition. 2020;123:1127-1137.

18. Kapil S, Duseja A, Sharma BK, Singla B, Chakraborti A, Das A, et al. Small intestinal bacterial overgrowth and toll-like receptor signaling in patients with non-alcoholic fatty liver disease. Journal of Gastroenterology and Hepatology. 2016;31:213-221.

19. Boursier J, Mueller O, Barret M, Machado M, Fizanne L, Araujo-Perez F, et al. The severity of nonalcoholic fatty liver disease is associated with gut dysbiosis and shift in the metabolic function of the gut microbiota. Hepatology. 2016;63:764-775.

20. Alonso C, Fernández-Ramos D, Varela-Rey M, Martínez-Arranz I, Navasa N, Van Liempd SM, et al. Metabolomic identification of subtypes of nonalcoholic steatohepatitis. Gastroenterology. 2017;152: 1449-61.e7.

21. Ye J, Lv L, Wu W, Li Y, Shi D, Fang D, et al. Butyrate protects mice against methionine-choline-deficient diet-induced non-alcoholic steatohepatitis by improving gut barrier function, attenuating inflammation and reducing endotoxin levels. Frontiers in Microbiology. 2018;9:1967.

22. Jäger R, Mohr AE, Carpenter KC, Kerksick CM, Purpura M, Moussa A, et al. International Society of Sports Nutrition Position Stand: Probiotics. Journal of the International Society of Sports Nutrition. 2019; 16:62.

23. Dahiya, Dinesh K, Puniya, Monica, Shandilya, Umesh K, et al. Gut Microbiota Modulation and Its Relationship with Obesity Using Prebiotic Fibers and Probiotics: A Review. Frontiers in Microbiology. 2017;8:563.

24. Porras D, Nistal E, Martínez-Flórez S, González-Gallego J, García-Mediavilla MV, Sánchez-Campos S. Intestinal microbiota modulation in obesity-related non-alcoholic fatty liver disease. Frontiers in Physiology. 2018;9:1813.

25. Quigley EMM. Prebiotics and Probiotics in Digestive Health. Clinical Gastroenterology and Hepatology. 2019;17:333-344.

26. Hou Q, Zhao F, Liu W, Lv R, Khine WWT, Han J, et al. Probiotic-directed modulation of gut microbiota is basal microbiome dependent. Gut Microbes. 2020;12:1-21:e1736974.

27. Arora T, Singh S, Sharma RK. Probiotics: interaction with gut microbiome and antiobesity potential. Nutrition. 2013;29:591-596.

28. Mokhtari Z, Gibson DL, Hekmatdoost A. Nonalcoholic Fatty Liver Disease, the Gut Microbiome, and Diet. Advances in Nutrition: An International Review Journal. 2017;8:240-252.

29. Eckburg PB, Eckburg PB, Bik EM, Bernstein CN, Purdom E, Dethlefsen L, et al. Diversity of the Human Intestinal Microbial Flora. Science. 2010;308:1635-1638.

30. Bäckhed F. Programming of host metabolism by the gut microbiota. Annals of Nutrition and Metabolism. 2011;58:44-52.

31. Lau LHS, Wong SH. Microbiota, obesity and NAFLD. Advances in Experimental Medicine and Biology. 2018;1061:111-125.

32. Bibbò S, Ianiro G, Dore MP, Simonelli C, Newton EE, Cammarota G. Gut microbiota as a driver of inflammation in nonalcoholic fatty liver disease. Mediators of inflammation. 2018;9321643.

33. Nobili V, Putignani L, Mosca A, Del Chierico F, Vernocchi P, Alisi A, et al. Bifidobacteria and lactobacilli in the gut microbiome of children with non-alcoholic fatty liver disease: which strains act as health players? Archives of medical science: Archives of Medical Science (AMS). 2018;14:81.

34. Kim H-N, Joo E-J, Cheong HS, Kim Y, Kim H-L, Shin H, et al. Gut Microbiota and Risk of Persistent Nonalcoholic Fatty Liver Diseases. Journal of Clinical Medicine. 2019;56:369-376. 
35. Caussy C, Tripathi A, Humphrey G, Bassirian S, Singh S, Faulkner C, et al. A gut microbiome signature for cirrhosis due to nonalcoholic fatty liver disease. Nature Communications. 2019;10:1406.

36. Bastian WP, Hasan I, Lesmana CRA, Rinaldi I, Gani RA. Gut Microbiota Profiles in Nonalcoholic Fatty Liver Disease and Its Possible Impact on Disease Progression Evaluated with Transient Elastography: Lesson Learnt from 60 Cases. Case Reports in Gastroenterology. 2019;13:125-133.

37. Mouzaki M, Comelli EM, Arendt BM, Bonengel J, Fung SK, Fischer SE, et al. Intestinal microbiota in patients with nonalcoholic fatty liver disease. Hepatology. 2013;58:120-127.

38. Everard A, Belzer C, Geurts L, Ouwerkerk JP, Druart C, Bindels LB, et al. Cross-talk between Akkermansia muciniphila and intestinal epithelium controls diet-induced obesity. Proceedings of the National Academy of Sciences. 2013;110:9066-9071.

39. Jiang W, Wu N, Wang X, Chi Y, Zhang Y, Qiu X, et al. Dysbiosis gut microbiota associated with inflammation and impaired mucosal immune function in intestine of humans with non-alcoholic fatty liver disease. Scientific Reports. 2015;5:8096.

40. Xie G, Wang X, Liu P, Wei R, Chen W, Rajani C, et al. Distinctly altered gut microbiota in the progression of liver disease. Oncotarget. 2016;7:19355-19366.

41. Shen F, Zheng RD, Sun XQ, Ding WJ, Wang XY, Fan JG. Gut microbiota dysbiosis in patients with non-alcoholic fatty liver disease. Hepatobiliary and Pancreatic Diseases International. 2017;16:375-381.

42. Del Chierico F, Nobili, VP, et al. Gut microbiota profiling of pediatric nonalcoholic fatty liver disease and obese patients unveiled by an integrated meta-omics-based approach. Hepatology. 2017;65:451-464.

43. SMB, Duarte JT,Stefano L, Miele, et al. Gut microbiome composition in lean patients with NASH is associated with liver damage independent of caloric intake: A prospective pilot study. Nutrition, Metabolism and Cardiovascular Diseases. 2018;28:369-384.

44. Caussy C, Hsu C, Lo MT, Liu A, Bettencourt R, Ajmera VH, et al. Link between gut-microbiome derived metabolite and shared gene-effects with hepatic steatosis and fibrosis in NAFLD. Hepatology. 2018;24:1070-1080

45. Ponziani FR, Bhoori S, Castelli C, Putignani L, Rivoltini L, Del Chierico F, et al. Hepatocellular Carcinoma Is Associated With Gut Microbiota Profile and Inflammation in Nonalcoholic Fatty Liver Disease. Hepatology. 2019;69:107-120.

46. Yun Y, Kim HN, Lee Ej, Ryu S, Chang Y, Shin H, et al. Fecal and blood microbiota profiles and presence of nonalcoholic fatty liver disease in obese versus lean subjects. PLoS ONE. 2019;14:e0213692

47. Brandl K, Kumar V, Eckmann L. Gut-liver axis at the frontier of host-microbial interactions. American Journal of Physiology-Gastrointestinal and Liver Physiology. 2017;312:G413-G419.

48. Wang B, Yao M, Lv L, Ling Z, Li L. The Human Microbiota in Health and Disease. Engineering. 2017;3:71-82.

49. Gupta H, Youn GS, Shin MJ, Suk KT. Role of Gut Microbiota in Hepatocarcinogenesis. Microorganisms. 2019;7:121.

50. Perumpail B, Li A, John N, The Therapeutic Implications of the Gut M, Probiotics in Patients with Nafldsallam S, Shah N, et al. The Therapeutic Implications of the Gut Microbiome and Probiotics in Patients with NAFLD. Diseases. 2019;7:27

51. Schnabl B, Brenner DA. Interactions between the intestinal microbiome and liver diseases. Gastroenterology. 2014;146:1513-1524.

52. Tripathi A, Debelius J, Brenner DA, Karin M, Loomba R, Schnabl B, et al. The gut-liver axis and the intersection with the microbiome. Nature Reviews Gastroenterology \& Hepatology. 2018;15:397-411.

53. Rao SSC, Bhagatwala J. Small Intestinal Bacterial Overgrowth: Clinical Features and Therapeutic Management. Clinical and Translational Gastroenterology. 2019;10:e00078.

54. Augustyn M, Grys I, Kukla M. Small intestinal bacterial overgrowth and nonalcoholic fatty liver disease. Clinical and Experimental Hepatology. 2019;5:1.

55. Marra F, Svegliati-Baroni G. Lipotoxicity and the gut-liver axis in NASH pathogenesis. Journal of Hepatology. 2018;68:280-295

56. Stefan N, Häring HU, Cusi K. Non-alcoholic fatty liver disease: causes, diagnosis, cardiometabolic consequences, and treatment strategies. The Lancet Diabetes \& Endocrinology. 2019;7(4):1-12.

57. Wang $\mathrm{W}$, Shi LP, Shi L, Xu L. Efficacy of probiotics on the treatment of non-alcoholic fatty liver disease. Chinese Journal of Internal Medicine. 2018;57:101-106.

58. Roh YS, Seki E. Toll-like receptors in alcoholic liver disease, non-alcoholic steatohepatitis and carcinogenesis. Journal of Gastroenterol Hepatology. 2013;28:38-42.

59. Wang R, Li H, Yang X, Xue X, Deng L, Shen J, et al. Genetically obese human gut microbiota induces liver steatosis in germ-free mice fed on normal diet. Frontiers in Microbiology. 2018;9:1-12.

60. Chiu CC, Ching YH, Li YP, Liu JY, Huang YT, Huang YW, et al. Nonalcoholic fatty liver disease is exacerbated in high-fat diet-fed gnotobiotic mice by colonization with the gut microbiota from patients with nonalcoholic steatohepatitis. Nutrients. 2017;9(11):1220.

61. Glavan TW, Gaulke CA, Santos Rocha C, Sankaran-Walters S, Hirao LA, Raffatellu M, et al. Gut immune dysfunction through impaired innate pattern recognition receptor expression and gut microbiota dysbiosis in chronic SIV infection. Mucosal Immunology. 2016;9:677-688
62. Federico A, Dallio M, Godos J, Loguercio C, Salomone F. Targeting gut-liver axis for the treatment of nonalcoholic steatohepatitis: translational and clinical evidence. Translational Research. 2016;167:116-124.

63. Assimakopoulos SF, Tsamandas AC, Tsiaoussis GI, Karatza E, Zisimopoulos D, Maroulis I, et al. Intestinal mucosal proliferation, apoptosis and oxidative stress in patients with liver cirrhosis. Annals of Hepatology. 2015;12:301-307.

64. Hendrikx T, Schnabl B. Antimicrobial proteins: Intestinal guards to protect against liver disease. Journal of Gastroenterology. 2019;54:209-217.

65. Rahman K, Desai C, Iyer SS, Thorn NE, Kumar P, Liu Y, et al. Loss of Junctional Adhesion Molecule A Promotes Severe Steatohepatitis in Mice on a Diet High in Saturated Fat, Fructose, and Cholesterol. Gastroenterology. 2016;151:733-741.

66. Nier A, Engstler AJ, Maier IB, Bergheim I. Markers of intestinal permeability are already altered in early stages of non-alcoholic fatty liver disease: Studies in children. PLoS ONE. 2017;12:e0183282.

67. Ponziani FR, Bhoori S, Castelli C, Putignani L, Rivoltini L, Del Chierico F, et al. Hepatocellular carcinoma is associated with gut microbiota profile and inflammation in nonalcoholic fatty liver disease. Hepatology. 2019;69:107-120.

68. Wahlström A, Sayin SI, Marschall HU, Bäckhed F. Intestinal Crosstalk between Bile Acids and Microbiota and Its Impact on Host Metabolism. Cell Metabolism. 2016;24:41-50.

69. Chávez-Talavera O, Tailleux A, Lefebvre P, Staels B. Bile Acid Control of Metabolism and Inflammation in Obesity, Type 2 Diabetes, Dyslipidemia, and Nonalcoholic Fatty Liver Disease. Gastroenterology. 2017;152:1679-1694.e3.

70. Arab JP, Karpen SJ, Dawson PA, Arrese M, Trauner M. Bile acids and nonalcoholic fatty liver disease: Molecular insights and therapeutic perspectives. Hepatology. 2017;65:350-362.

71. Wahlström A, Kovatcheva-Datchary P, Ståhlman M, Bäckhed F, Marschall HU. Crosstalk between Bile Acids and Gut Microbiota and Its Impact on Farnesoid X Receptor Signalling. Digestive Diseases. 2017;35:246-250.

72. Ridlon JM, Harris SC, Bhowmik S, Kang DJ, Hylemon PB. Consequences of bile salt biotransformations by intestinal bacteria. Gut Microbes. 2016;7:22-39.

73. Yokota A, Fukiya S, Islam KBMS, Ooka T, Ogura Y, Hayashi T, et al. Is bile acid a determinant of the gut microbiota on a high-fat diet? Gut Microbes. 2014:3:455-459.

74. Lau LH, Wong SH. Microbiota, obesity and NAFLD. Advances in Experimental Medicine and Biology. 2018;1061:111-125.

75. Thomas C, Gioiello A, Noriega L, Strehle A, Oury J, Rizzo G, et al. TGR5-Mediated Bile Acid Sensing Controls Glucose Homeostasis. Cell Metabolism. 2009;10:167e177.

76. Mouzaki M, Wang AY, Bandsma R, Comelli EM, Arendt BM, Zhang L, et al. Bile acids and dysbiosis in non-alcoholic fatty liver disease. PLoS ONE. 2016;11:e0151829.

77. Chow MD, Lee YH, Guo GL. The role of bile acids in nonalcoholic fatty liver disease and nonalcoholic steatohepatitis. Molecular Aspects of Medicine. 2017;56:34-44

78. Parséus A, Sommer N, Sommer F, Caesar R, Molinaro A, Stahlman M, et al. Microbiota-induced obesity requires farnesoid $X$ receptor. Gut. 2017;66:429-437.

79. Torres J, Palmela C, Brito H, Bao X, Ruiqi H, Moura-Santos P, et al. The gut microbiota, bile acids and their correlation in primary sclerosing cholangitis associated with inflammatory bowel disease. United European Gastroenterology Journal. 2018;6:112-122.

80. Bechmann LP, Hannivoort RA, Gerken G, Hotamisligil GS, Trauner M, Canbay A. The interaction of hepatic lipid and glucose metabolism in liver diseases. Journal of Hepatology. 2012;56:952-964.

81. Deutschmann K, Reich M, Klindt C, Dröge C, Spomer L, Häussinger D, et al. Bile acid receptors in the biliary tree: TGR5 in physiology and disease. Biochimica et Biophysica Acta (BBA)-Molecular Basis of Disease. 2018;1864:1319-1325.

82. Cani PD. Metabolism in 2013: the gut microbiota manages host metabolism. Nature Reviews Endocrinology. 2014;10:74-76.

83. Ma J, Zhou Q, Li H. Gut microbiota and nonalcoholic fatty liver disease: Insights on mechanisms and therapy. Nutrients. 2017;9(10):1124.

84. Cole LK, Vance JE, Vance DE. Phosphatidylcholine biosynthesis and lipoprotein metabolism. Biochimica et Biophysica Acta (BBA)-Molecular and Cell Biology of Lipids. 2012;1821:754-761.

85. Sherriff JL, O'Sullivan TA, Properzi C, Oddo J-L, Adams LA. Choline, its potential role in nonalcoholic fatty liver disease, and the case for human and bacterial genes. Advances in Nutrition. 2016; 7:5-13.

86. Stremmel W, Schmidt KV, Schuhmann V, Kratzer F, Garbade SF, Langhans $\mathrm{CD}$, et al. Blood trimethylamine-n-oxide originates from microbiota mediated breakdown of phosphatidylcholine and absorption from small intestine. PLoS ONE. 2017;12:e170742.

87. Ierardi E, Sorrentino C, Principi M, Giorgio F, Losurdo G, Di Leo A. Intestinal microbial metabolism of phosphatidylcholine: a novel insight in the cardiovascular risk scenario. Hepatobiliary Surgery and Nutrition. 2015;4:289.

88. Kirpich IA, Solovieva NV, Leikhter SN, Shidakova NA, Lebedeva OV, Sidorov PI, et al. Probiotics restore bowel flora and improve liver enzymes in human alcohol-induced liver injury: a pilot study. Alcohol. 2008;42:675-682.

89. Leung C, Rivera L, Furness JB, Angus PW. The role of the gut microbiota in NAFLD. Nature Reviews Gastroenterology \& Hepatology. 2016;13:412-425.

90. Tosello-Trampont A-C, Landes SG, Nguyen V, Novobrantseva TI, Hahn YS. Kuppfer cells trigger nonalcoholic steatohepatitis development in 
diet-induced mouse model through tumor necrosis factor- $\alpha$ production. Journal of Biological Chemistry. 2012;287:40161-40172.

91. Fennema D, Phillips IR, Shephard EA. Trimethylamine and trimethylamine $\mathrm{N}$-oxide, a flavin-containing monooxygenase 3 (FMO3)-mediated host-microbiome metabolic axis implicated in health and disease. Drug Metabolism and Disposition. 2016;44:1839-1850.

92. Mehedint MG, Zeisel SH. Choline's role in maintaining liver function: New evidence for epigenetic mechanisms. Current Opinion in Clinical Nutrition \& Metabolic Care. 2013;16:339-345.

93. Nowiński A, Ufnal M. Trimethylamine N-oxide: a harmful, protective or diagnostic marker in lifestyle diseases? Nutrition. 2018;46:7-12.

94. Gogiashvili M, Edlund K, Gianmoena K, Marchan R, Brik A, Andersson JT, et al. Metabolic profiling of ob/ob mouse fatty liver using HR-MAS 1H-NMR combined with gene expression analysis reveals alterations in betaine metabolism and the transsulfuration pathway. Analytical and Bioanalytical Chemistry. 2017;409:1591-1606

95. Chen YM, Liu Y, Zhou RF, Chen XL, Wang C, Tan XY, et al. Associations of gut-flora-dependent metabolite trimethylamine-N-oxide, betaine and choline with non-alcoholic fatty liver disease in adults. Scientific Reports. 2016;6:19076.

96. Brandl K, Schnabl B. Intestinal microbiota and nonalcoholic steatohepatitis. Current Opinion in Gastroenterology. 2017;33:128-133.

97. Nicoletti A, Ponziani FR, Biolato M, Valenza V, Marrone G, Sganga G, et al. Intestinal permeability in the pathogenesis of liver damage: From non-alcoholic fatty liver disease to liver transplantation. World journal of Gastroenterology. 2019;25:4814

98. Engstler AJ, Aumiller T, Degen C, Dürr M, Weiss E, Maier IB, et al. Insulin resistance alters hepatic ethanol metabolism: studies in mice and children with non-alcoholic fatty liver disease. Gut. 2016; 65:1564-1571.

99. Ma J, Zhou Q, Li H. Gut microbiota and nonalcoholic fatty liver disease: insights on mechanisms and therapy. Nutrients. 2017:9:1124.

100. Mir H, Meena AS, Chaudhry KK, Shukla PK, Gangwar R, Manda B, et al. Occludin deficiency promotes ethanol-induced disruption of colonic epithelial junctions, gut barrier dysfunction and liver damage in mice. Biochimica et Biophysica Acta - General Subjects. 2016;1860:765-774.

101. Hartmann P, Seebauer CT, Mazagova M, Horvath A, Wang L, Llorente C, et al. Deficiency of intestinal mucin-2 protects mice from diet-induced fatty liver disease and obesity. American Journal of Physiology-Gastrointestinal and Liver Physiology. 2016;10:G310-G322.

102. Cresci GA, Glueck B, McMullen MR, Xin W, Allende D, Nagy LE. Prophylactic tributyrin treatment mitigates chronic-binge ethanol-induced intestinal barrier and liver injury. Journal of Gastroenterology and Hepatology (Australia). 2017;32:1587-1597.

103. Ansari RA, Husain K, Rizvi SAA. Role of transcription factors in steatohepatitis and hypertension after ethanol: The epicenter of metabolism. Biomolecules. 2016;6:29.

104. Hamarneh SR, Kim BM, Kaliannan K, et al. Intestinal Alkaline Phosphatase Attenuates Alcohol-Induced Hepatosteatosis in Mice. Digestive Diseases and Sciences. 2017;62:2021-2034.

105. Morrison DJ, Preston T. Formation of short chain fatty acids by the gut microbiota and their impact on human metabolism. Gut Microbes. 2016;7:189-200.

106. Valdes AM, Walter J, Segal E, Spector TD. Role of the gut microbiota in nutrition and health. BMJ (Online). 2018;361:k2179.

107. Weitkunat K, Schumann S, Nickel D, Kappo KA, Petzke KJ, Kipp AP, et al. Importance of propionate for the repression of hepatic lipogenesis and improvement of insulin sensitivity in high-fat diet-induced obesity. Molecular Nutrition \& Food Research. 2016;60:2611-2621.

108. He M, Shi B. Gut microbiota as a potential target of metabolic syndrome: The role of probiotics and prebiotics. Cell Biosci. Cell \& Bioscience. 2017;7:54.

109. Qin N, Yang F, Li A, Prifti E, Chen Y, Shao L, et al. Alterations of the human gut microbiome in liver cirrhosis. Nature. 2014:513:59-64.

110. Canfora EE, Jocken JW, Blaak EE. Short-chain fatty acids in control of body weight and insulin sensitivity. Nature Reviews Endocrinology. 2015;11:577.

111. Aragonès G, González-García S, Aguilar C, Richart C, Auguet T. Gut Microbiota-Derived Mediators as Potential Markers in Nonalcoholic Fatty Liver Disease. BioMed Research International. 2019;1-10.

112. Zhou D, Pan Q, Xin FZ, et al. Sodium butyrate attenuates high-fat dietinduced steatohepatitis in mice by affecting GLP-1 and gut microbiota. Journal of Gastroenterology and Hepatology (Australia). 2016;23:60-75.

113. Baumann A, Jin CJ, Brandt A, Sellmann C, Nier A, Burkard M, et al. Oral supplementation of sodium butyrate attenuates the progression of non-alcoholic steatohepatitis. Nutrients. 2020;12:951.

114. Vos T, Abajobir AA, Abbafati C, Abbas KM, Abate KH, Abd-Allah F, et al. Global, regional, and national incidence, prevalence, and years lived with disability for 328 diseases and injuries for 195 countries, 1990-2016: A systematic analysis for the Global Burden of Disease Study 2016. The Lancet. 2017;390:10100:1211-1259.

115. Martinez KB, Leone V, Chang EB. Microbial metabolites in health and disease: Navigating the unknown in search of function. Journal of Biological Chemistry. 2017;292:8553-8559.

116. Zhou D, Chen Y-W, Zhao Z-H, Yang R-X, Xin F-Z, Liu X-L, et al. Sodium butyrate reduces high-fat diet-induced non-alcoholic steatohepatitis through upregulation of hepatic GLP-1R expression. Experimental \& Molecular Medicine. 2018;50:1-12
117. Armstrong MJ, Hull D, Guo K, Barton D, Hazlehurst JM, Gathercole LL, et al. Glucagon-like peptide 1 decreases lipotoxicity in non-alcoholic steatohepatitis. Journal of Hepatology. 2016;64:399-408.

118. Joseph N, Vasodavan K, Saipudin NA, Yusof BNM, Kumar S, Nordin SA. Gut microbiota and short-chain fatty acids (SCFAs) profiles of normal and overweight school children in Selangor after probiotics administration. Journal of Functional Foods. 2019;57:103-111.

119. Dalile B, Van Oudenhove L, Vervliet B, Verbeke K. The role of short-chain fatty acids in microbiota-gut-brain communication. Nature Reviews Gastroenterology \& Hepatology. 2019;16:461-478.

120. Rooks MG, Garrett WS. Gut microbiota, metabolites and host immunity. Nature Reviews Immunology. 2016;16:341-352.

121. Lopetuso LR, Petito V, Graziani C, Schiavoni E, Sterbini FP, Poscia A, et al. Gut microbiota in health, diverticular disease, irritable bowel syndrome, and inflammatory bowel diseases: time for microbial marker of gastrointestinal disorders. Digestive Diseases. 2018;36:56-65.

122. Yamashiro Y. Gut Microbiota in Health and Disease. Annals of Nutration and Metabolism.2018;71:242-246.

123. Chen J, Thomsen M, Vitetta L. Interaction of gut microbiota with dysregulation of bile acids in the pathogenesis of nonalcoholic fatty liver disease and potential therapeutic implications of probiotics. Journal of Cellular Biochemistry. 2019;120:2713-2720.

124. Liang Y, Liang S, Zhang Y, et al. Oral Administration of Compound Probiotics Ameliorates HFD-Induced Gut Microbe Dysbiosis and Chronic Metabolic Inflammation via the G Protein-Coupled Receptor 43 in Non-alcoholic Fatty Liver Disease Rats. Probiotics and Antimicrobial Proteins. 2018;11:175-185.

125. Duseja A, Acharya SK, Mehta M, Chhabra S, Rana S, Das A, et al. High potency multistrain probiotic improves liver histology in non-alcoholic fatty liver disease (NAFLD): a randomised, double-blind, proof of concept study. BMJ Open Gastroenterology. 2019;6:e000315.

126. Saltzman ET, Palacios T, Thomsen M, Vitetta L. Intestinal microbiome shifts, dysbiosis, inflammation, and non-alcoholic fatty liver disease. Frontiers in Microbiology. 2018;9:61.

127. Moossavi S, Miliku K, Sepehri S, Khafipour E, Azad MB. The prebiotic and probiotic properties of human milk: implications for infant immune development and pediatric asthma. Frontiers in Pediatrics. 2018;6:197.

128. Cho MS, Kim SY, Suk KT, Kim BY. Modulation of gut microbiome in nonalcoholic fatty liver disease: pro-, pre-, syn-, and antibiotics. Journal of Microbiology. 2018;56:855-867.

129. AmmarCota G, Bibbò S, Newton EE, Simonelli C, Ianiro G, Dore MP. Gut Microbiota as a Driver of Inflammation in Nonalcoholic Fatty Liver Disease. Mediators of Inflammation. 2018;1-7.

130. Liao SF, Nyachoti M. Using probiotics to improve swine gut health and nutrient utilization. Animal Nutrition. 2017;3:331-343.

131. Gibson GR, Hutkins RW, Sanders ME, Prescott SL, Reimer RA, Salminen SI, et al. The International Scientific Association for Probiotics and Prebiotics (ISAPP) consensus statement on the definition and scope of prebiotics. Nature Reviews Gastroenterology and Hepatology. 2017;14:491-502

132. de Melo Pereira GV, de Oliveira Coelho B, Magalhães Júnior AI, Thomaz-Soccol V, Soccol CR. How to select a probiotic? A review and update of methods and criteria. Biotechnology Advances. 2018;36:2060-2076.

133. Jang, Hye RP, Hyun-Jun,Kang, Dongwon, Chung. et al. A protective mechanism of probiotic Lactobacillus against hepatic steatosis via reducing host intestinal fatty acid absorption. Experimental and Molecular Medicine. 2019;51:1-14

134. Zhao Z, Wang C, Zhang L, Zhao Y, Duan C, Zhang X, et al. Lactobacillus plantarum NA136 improves the non-alcoholic fatty liver disease by modulating the AMPK/Nrf2 pathway. Applied Microbiology and Biotechnology. 2019:103:5843-5850.

135. Song W, Song C, Shan Y, Lu W, Zhang J, Hu P, et al. The antioxidative effects of three Lactobacilli on high-fat diet induced obese mice. RSC Advances. 2016;6:65808-65815.

136. Yan Y, Chunyan L, Shimin Z, Xinxu W, Jinling W, Heping Z, et al. Probiotic Bifidobacterium lactis $\mathrm{V} 9$ attenuates hepatic steatosis and inflammation in rats with non-alcoholic fatty liver disease. AMB Express (springer Open). 2020;10:101

137. Iacono A, Raso GM, Canani RB, Calignano A, Meli R. Probiotics as an emerging therapeutic strategy to treat NAFLD: Focus on molecular and biochemical mechanisms. Journal of Nutritional Biochemistry. 2011;22:699-711.

138. Kim DH, Kim H, Jeong D, et al. Kefir alleviates obesity and hepatic steatosis in high-fat diet-fed mice by modulation of gut microbiota and mycobiota: targeted and untargeted community analysis with correlation of biomarkers. Journal of Nutritional Biochemistry. 2017;44:35-43.

139. Xue L, He J, Gao N, Lu X, Li M, Wu X, et al. Probiotics may delay the progression of nonalcoholic fatty liver disease by restoring the gut microbiota structure and improving intestinal endotoxemia. Scientific Reports. 2017;7:45176.

140. Yvonne R, Gyöngyi B, Anke C, Veronika E, Ina B, Andreas S, et al. Lactobacillus rhamnosus GG protects against non-alcoholic fatty liver disease in mice. PloS ONE. 2014;9:e80169.

141. Park E-J, Lee Y-S, Kim SM, Park G-S, Lee YH, Jeong DY, et al. Beneficial Effects of Lactobacillus plantarum Strains on Non-Alcoholic Fatty Liver Disease in High Fat/High Fructose Diet-Fed Rats. Nutrients. 2020;12:542. 
142. Briskey D, Heritage M, Jaskowski LA, Peake J, Gobe G, Subramaniam VN, et al. Probiotics modify tight-junction proteins in an animal model of nonalcoholic fatty liver disease. Therapeutic Advances in Gastroenterology. 2016;9:463-472.

143. Gao J, Li Y, Wan Y, Hu T, Liu L, Yang S, et al. A novel postbiotic from Lactobacillus rhamnosus GG with a beneficial effect on intestinal barrier function. Frontiers in microbiology. 2019;10:477.

144. Kong C, Gao R, Yan X, Huang L, Qin H. Probiotics improve gut microbiota dysbiosis in obese mice fed a high-fat or high-sucrose diet. Nutrition. 2019;60:175-184.

145. Hsieh F-C, Wu C-S, Lee C-L, Chai C-Y, Chen W-T, Lu Y-C. Oral administration of Lactobacillus reuteri GMNL-263 improves insulin resistance and ameliorates hepatic steatosis in high fructose-fed rats. Nutrition \& Metabolism. 2013;10:35.

146. Bubnov RV, Babenko LP, Lazarenko LM, Mokrozub VV, Demchenko OA, Nechypurenko OV, et al. Comparative study of probiotic effects of Lactobacillus and Bifidobacteria strains on cholesterol levels, liver morphology and the gut microbiota in obese mice. EPMA Journal. 2017;:8(4):357-376.

147. Lu Mei, Youcai T, Ming L, Pingchang Y, Zhiqiang L, Jieli Y, Pengyuan Z. Co-administration of cholesterol-lowering probiotics and anthraquinone from Cassia obtusifolia L. Ameliorate non-alcoholic fatty liver. PLoS ONE. 2015;10:e0138078.

148. Wang G, Jiao T, Xu Y, Li D, Si Q, Hao J, et al. Bifidobacterium adolescentis and Lactobacillus rhamnosus alleviate non-alcoholic fatty liver disease induced by a high-fat, high-cholesterol diet through modulation of different gut microbiota-dependent pathways. Food \& Function. 2020;7:1-23.

149. Famouri F, Shariat Z, Hashemipour M, Keikha M, Kelishadi R. Effects of probiotics on nonalcoholic fatty liver disease in obese children and adolescents. Journal of Pediatric Gastroenterology and Nutrition. 2017;64:413-417.

150. Malaguarnera M, Vacante M, Antic T, Giordano M, Chisari G, Acquaviva R, et al. Bifidobacterium longum with fructo-oligosaccharides in patients with non alcoholic steatohepatitis. Digestive Diseases and Sciences. 2012;57:545-553.

151. Lavekar AS, Raje DV, Manohar T, Lavekar AA. Role of Probiotics in the Treatment of Nonalcoholic FattyLiver Disease: A Meta-analysis. Euroasian Journal of Hepato-Gastroenterology. 2017; 7:130-137.

152. Loman BR, Hernández-Saavedra D, An R, Rector RS. Prebiotic and probiotic treatment of nonalcoholic fatty liver disease: A systematic review and meta-analysis. Nutrition Reviews. 2018;76:822-839.

153. Suez J, Zmora N, Segal E, Elinav E. The pros, cons, and many unknowns of probiotics. Nature Medicine. 2019;25:716-729.

154. McNaught CE, Woodcock NP, MacFie J, Mitchell CJ. A prospective randomised study of the probiotic Lactobacillus plantarum $299 \mathrm{~V}$ on indices of gut barrier function in elective surgical patients. Gut. 2002;51:827-831.

155. Chalasani N, Younossi Z, Lavine JE, Diehl AM, Brunt EM, Cusi K, et al. The diagnosis and management of non-alcoholic fatty liver disease: Practice Guideline by the American Association for the Study of Liver Diseases, American College of Gastroenterology, and the American Gastroenterological Association. Hepatology. 2012;107:811-826.

156. Bakhshimoghaddam F, Shateri K, Sina M, Hashemian M, Alizadeh M. Daily consumption of synbiotic yogurt decreases liver steatosis in patients with nonalcoholic fatty liver disease: a randomized controlled clinical trial. The Journal of Nutrition. 2018;148:1276-1284

157. Vajro P, Mandato C, Licenziati MR, Franzese A, Vitale DF, Lenta S, et al. Effects of Lactobacillus rhamnosus strain gg in pediatric obesity-related liver disease. Journal of Pediatric Gastroenterology and Nutrition. 2011:52:740-743.

158. Nabavi S, Rafraf M, Somi M, Homayouni-Rad A, Asghari-Jafarabadi M. Effects of probiotic yogurt consumption on metabolic factors in individuals with nonalcoholic fatty liver disease. Journal of Dairy Science. 2014;97:7386-7393.

159. Ahn SB, Jun DW, Kang B-K, Lim JH, Lim S, Chung M-J. Randomized, double-blind, placebo-controlled study of a multispecies probiotic mixture in nonalcoholic fatty liver disease. Scientific Reports. 2019;9:1-9. 